This is the peer reviewed version of the following article:

Yann Hendrik Simsont, Peter Gerlinger, High order numerical simulation of the thermal load on a lobed strut injector for scamjet applications, International Journal for Numerical Methods in Fluids 82 (2016) 417-436,

which has been published in final form at

http://onlinelibrary.wiley.com/doi/10.1002/fld.4224/full

http://dx.doi.org/10.1002/fld.4224

This article may be used for non-commercial purposes in accordance with Wiley Terms and Conditions for Self-Archiving. 


\title{
High Order Numerical Simulation of the Thermal Load on a Lobed Strut Injector for Scramjet Applications
}

\author{
Yann Hendrik Simsont and Peter Gerlinger* \\ Institut für Verbrennungstechnik der Luft- und Raumfahrt, Universität Stuttgart, Pfaffenwaldring 38-40, 70569 Stuttgart
}

\begin{abstract}
SUMMARY
In this paper the thermal load on an actively cooled lobed strut injector for scramjet (supersonic combustion ramjet) applications is investigated numerically. This requires coupled simulations of the strut internal and external flow fields together with the heat conduction in the solid injector body. In order to achieve a fast mixing, the lobed strut is positioned at the channel axis to inject hydrogen into the core of a Mach 3 air stream. There it is exposed to the extremely high temperatures of the high speed flow. While the external air and hydrogen flows are supersonic the strut internal hydrogen flow is mainly subsonic, in some regions at very low Mach numbers. To enable a simulation of the internal flow field which ranges from very low to very high Mach numbers (approximately Mach 2.25 at the nozzle exit), a preconditioning technique is employed. The compressible finite-volume scheme uses a spatially fourth order MLP (multi-dimensional limiting process) $[1,2]$ discretization which is used here for a first time to simulate a geometrically and fluid mechanically highly complex problem. It will be demonstrated that besides its high accuracy the MLP scheme is numerically stable even in case of demanding practical applications. The coupled simulation of the lobed strut injector delivers unique insight into the flow phenomena inside and outside the strut, the heat fluxes, the temperature distribution in the solid material, the required hydrogen mass flux with respect to cooling requirements and details concerning the conditions at the exit of the injector. Copyright (c) 2015 John Wiley \& Sons, Ltd.

Received ..
\end{abstract}

KEY WORDS: Scramjet, lobed strut injector, injector cooling, thermal impact, conjugate heat transfer, preconditioning, MLP, high order discretization

Copyright (c) 2015 John Wiley \& Sons, Ltd.

Prepared using fldauth.cls [Version: 2010/05/13 v2.00] 


\section{INTRODUCTION}

In order to achieve a short length for a scramjet combustion chamber, rapid mixing of fuel and air is essential. Two main concepts for fuel placement can be distinguished: wall $[3,4,5,6,7]$ and strut injectors $[8,9,10,11]$. Wall injectors supply the fuel through the combustor walls or through wallmounted ramps either in crossflow or at a chosen angle to the main flow. In contrast, strut injectors are mounted in the center of the combustor and inject the fuel directly into the core of the air flow. This is particularly advantageous for large combustors where the penetration of a wall-injected reacting fuel jet may not be sufficient. As the fuel usually exits a strut injector in flow direction, it causes no blockage of the main flow. In fact, extra momentum is added by the fuel which increases the thrust of the scramjet engine. Lobe shaped struts $[12,13,14]$ can be used to enhance the mixing of fuel and air by creation of streamwise vorticity. However, in contrast to pure wall injection, strut injectors will cause total pressure losses, even if no fuel is injected. Another disadvantage of strut injectors is the cooling challenge: in particular the leading edge suffers from high thermal loads due to the high total temperatures at supersonic or hypersonic flight. In the investigated test case (Mach 8 flight conditions) the static temperature inside the combustor (at Mach 3) is $1160 \mathrm{~K}$, which corresponds to a total temperature of approximately $3250 \mathrm{~K}$. If the fuel is used for internal cooling, the strut injection may never be switched off completely and the minimal required fuel mass flow has to be determined for the chosen operating conditions.

There are many different concepts for strut injectors $[12,15,16,10]$ which differ in geometrical details as well as in their losses in total pressure and mixing efficiencies. Despite the fact that cooling is a great challenge, up to now no investigation concerning the thermal load on a strut is known to the authors (with exception of previous conference papers from the authors). In [17] the cooling of a pylon injector by films is investigated, however, without heat conduction in the material or considering the cooling effect of the hydrogen injected. The strut geometry used in the present investigation [12] has proven to work efficiently and reliably in a connected pipe test facility

\footnotetext{
${ }^{*}$ Correspondence to: peter Gerlinger, Institut für Verbrennungstechnik der Luft- und Raumfahrt, Universität Stuttgart, Pfaffenwaldring 38-40, 70569 Stuttgart. E-mail: peter.gerlinger@dlr.de 
$[18,19]$. Even in long time experiment this strut showed no significant thermal damage. However, the total temperature achieved in these experiments have been significantly lower than in real flight (approximately $1400 \mathrm{~K}$ compared to $3200 \mathrm{~K}$ and more). As continuously operating test facilities do not reach the relevant level of total temperature and in hot-shot wind tunnels (which reach the required temperatures) the measurement times are too short to investigate the thermal impact on strut injectors, this problem can not be studied by ground experiments. Therefore, numerical simulations of strut injector cooling are of high importance. This requires reliable and good validated numerical schemes which have to cover the full range from incompressible low Mach number flows up to compressible high speed conditions.

In order to investigate the interrelation between the hydrogen flow field inside the strut, the external flow field around it, and the heat conduction in the solid injector body, a coupled simulation of the three domains is required. Such a complex and computationally intensive numerical simulation is presented for realistic scramjet flight conditions. In detail the aims of this simulation are to

1. investigate the thermal impact and temperature distribution in the solid strut,

2. evaluate the flow management inside the strut,

3. evaluate the cooling of the leading edge (here a blunt tip configuration is chosen, therefore the resulting shock wave pattern is also of interest),

4. propose a hydrogen mass flux which is capable to sufficiently cool the strut for the used operating conditions,

5. determine more realistic hydrogen inflow conditions for combustor simulations that do not include the interior of the injector and the strut material.

Furthermore the preconditioning technique and the new high order spatial discretization technique $\operatorname{MLP}^{l d}$ (multi-dimensional limiting process, low diffusion) [20,2] will be presented. It will be shown that highly accurate results are possible at low additional computational cost.

To investigate the thermal impact on the lobed strut injector three different domains are simulated separately, the 
1. supersonic external gas phase flow (air and hydrogen, air is simulated as a mixture of oxygen and nitrogen),

2. mainly subsonic internal gas phase flow through the injector (hydrogen), and

3. heat conduction in the solid injector material (copper).

These steady-state simulations are coupled by an exchange of heat flux information at the wall boundaries after a defined number of iterations. Two in-House codes are used for these simulations: TASCOM3D (Turbulent All Speed Combustion Multigrid solver) [21, 12, 22, 2] for the gas flows and HeatEQ [23] for heat conduction in the solid. Both solvers are cell-centered finite-volumes schemes which use structured, multiblock grids. The numerical solutions are advanced in time.

\subsection{Gas phase}

The set of averaged equations to be solved for the gas flow is given in three-dimensional conservative form by

$$
\frac{\partial \mathbf{Q}_{\mathrm{c}}}{\partial t}+\frac{\partial\left(\mathbf{F}-\mathbf{F}_{\nu}\right)}{\partial x}+\frac{\partial\left(\mathbf{G}-\mathbf{G}_{\nu}\right)}{\partial y}+\frac{\partial\left(\mathbf{H}-\mathbf{H}_{\nu}\right)}{\partial z}=\mathbf{S}
$$

with the conservative variable vector

$$
\mathbf{Q}_{\mathrm{c}}=\left[\bar{\rho}, \bar{\rho} \widetilde{u}, \bar{\rho} \widetilde{v}, \bar{\rho} \widetilde{w}, \bar{\rho} \widetilde{E}, \bar{\rho} q, \bar{\rho} \omega, \bar{\rho} \widetilde{Y}_{i}\right]^{T} .
$$

Hereby, $\bar{\rho}$ denotes the Reynolds averaged density, $\widetilde{u}, \widetilde{v}$, and $\widetilde{w}$ the Favre averaged velocity components, $\widetilde{E}$ the total specific energy, and $\widetilde{Y}_{i}$ the species mass fractions for $i=1, \ldots, N_{k}-1$, where $N_{k}$ is the number of different species. Because combustion is not considered in the present study, the source vector

$$
\mathbf{S}=\left[0,0,0,0,0, S_{q}, S_{\omega}, 0\right]^{T}
$$

includes terms from turbulence modeling only. The vectors $\mathbf{F}, \mathbf{G}, \mathbf{H}$ describe the inviscid and the vectors $\mathbf{F}_{\nu}, \mathbf{G}_{\nu}, \mathbf{H}_{\nu}$ the viscous fluxes in $x$-, $y$-, and $z$-direction, respectively. For turbulence closure a low-Reynolds-number $q-\omega$ turbulence model [24] is employed with the turbulence 
variables $q=\sqrt{k}$ and $\omega=\epsilon / k$, which are formed connected to the turbulent kinetic energy $k$ and its dissipation rate $\epsilon$.

\subsection{Heat conduction in the solid}

To simulate heat conduction in the solid injector body the three-dimensional time-dependent energy equation

$$
\rho V c_{p} \frac{\partial T}{\partial t}+\oint_{S} \lambda \nabla T d S=0
$$

is solved, using Fourier's law to describe the heat fluxes. In this equation $V$ is the cell volume, $\rho$ the density, and $S$ the cell surface. The specific heat $c_{p}$ and the heat conductivity $\lambda$ are temperature dependent material properties for which polynomial functions are used. For the injector material copper the corresponding functions are deduced from data of Çengel [25].

Conjugate heat transfer At the boundaries to the internal and external gas phase flows the wall temperature $T_{\text {wall }}$ is calculated by equating the heat flux from the gas to the wall and the heat flux in the solid according to

$$
\lambda_{g} \frac{T_{g}-T_{\text {wall }}}{\Delta y_{g}}=\lambda_{s} \frac{T_{\text {wall }}-T_{s}}{\Delta y_{s}}
$$

The index ' $g$ ' denotes values of the gas phase, while ' $s$ ' represents values of the solid injector. $T_{g}$ and $T_{s}$ are the cell center values of the volumes adjacent to the boundary. The corresponding wall distances are represented by $\Delta y_{g}$ and $\Delta y_{s}$, respectively. Directly at the wall (at $y=0$ ) the heat fluxes in the gas phase are determined by conduction only and are calculated using the Fourier's law. This requires very fine near wall grids to accurately resolve the gas temperature profile in immediate proximity to the wall.

\section{NUMERICAL SOLVER FOR GAS PHASE}

For time integration of the original or preconditioned set of equations an implicit Lower-Upper Symmetric Gauß-Seidel (LU-SGS) algorithm [26, 27, 28] is implemented in TASCOM3D. In this 
paper only steady-state problems are investigated, which apply a first order temporal discretization. While for the purely subsonic test cases a sixth order central discretization may be employed for the inviscid fluxes, all supersonic (or at least partly supersonic) simulations are based on the upwind biased fourth order scheme. For interface flux calculation the $\mathrm{AUSM}^{+}$-up flux vector splitting method [29] is used. The calculation of the viscous fluxes is realized by central discretization. TASCOM3D is fully vectorized and parallelized using MPI [30]. Both the turbulent Prandtl and Schmidt numbers are assumed to be 0.7 .

\subsection{Preconditioning}

The convective eigenvalues of the system of equations (1) in $x$-direction are $\lambda_{x, 1,2}=\widetilde{u} \pm c$, and the multiple eigenvalue $\widetilde{u}$ with the speed of sound $c$. Consequently, for $M a \rightarrow 0$ the maximum and minimum eigenvalues of the system strongly diverge in magnitude and a condition number $K_{x} \rightarrow \infty$ is obtained. As a consequence convergence difficulties may arise if the flow velocity approaches zero. In order to rectify the stiffness of the set of governing equations the time derivatives are premultiplied by a preconditioning matrix $\Gamma$ that scales all convective eigenvalues of the system to the same order of magnitude. As this perturbs the time accuracy, a dual time stepping technique $[31,32]$ is applied and instead of Eq. (1)

$$
\frac{\partial \mathbf{Q}_{\mathrm{c}}}{\partial t}+\boldsymbol{\Gamma} \frac{\partial \mathbf{Q}_{\mathbf{p}}}{\partial \tau}+\frac{\partial\left(\mathbf{F}-\mathbf{F}_{\nu}\right)}{\partial x}+\frac{\partial\left(\mathbf{G}-\mathbf{G}_{\nu}\right)}{\partial y}+\frac{\partial\left(\mathbf{H}-\mathbf{H}_{\nu}\right)}{\partial z}=\mathbf{S}
$$

is solved. For the steady state simulations of this paper the inner iterations (and thus the first term in Eq. (4)) are not required and the solution is advanced in pseudo time until a steady state solution is obtained. Here $t$ is the physical time, $\tau$ a pseudo time, and the primitive variable vector is

$$
\mathbf{Q}_{\mathrm{p}}=\left[\bar{p}, \widetilde{u}, \widetilde{v}, \widetilde{w}, \widetilde{T}, q, \omega, \widetilde{Y}_{i}\right]^{T},
$$


using the pressure $\bar{p}$ instead of the density. The preconditioning matrix $\Gamma$ is given by

$$
\boldsymbol{\Gamma}=\left[\begin{array}{cccccccccc}
\frac{1}{\beta} & 0 & 0 & 0 & -\frac{\bar{\rho}}{\widetilde{T}} & 0 & 0 & \frac{\partial \bar{\rho}}{\partial \widetilde{Y}_{1}} & \ldots & \frac{\partial \bar{\rho}}{\partial \widetilde{Y}_{N_{k}-1}} \\
\frac{\widetilde{u}}{\beta} & \bar{\rho} & 0 & 0 & -\frac{\bar{\rho} \widetilde{u}}{\widetilde{T}} & 0 & 0 & \widetilde{u} \frac{\partial \bar{\rho}}{\partial \widetilde{Y}_{1}} & \ldots & \widetilde{u} \frac{\partial \bar{\rho}}{\partial \widetilde{Y}_{N_{k}-1}} \\
\frac{\widetilde{v}}{\beta} & 0 & \bar{\rho} & 0 & -\frac{\bar{\rho} \widetilde{v}}{\widetilde{T}} & 0 & 0 & \widetilde{v} \frac{\partial \bar{\rho}}{\partial \widetilde{Y}_{1}} & \ldots & \widetilde{v} \frac{\partial \bar{\rho}}{\partial \widetilde{Y}_{N_{k}-1}} \\
\frac{\widetilde{w}}{\beta} & 0 & 0 & \bar{\rho} & -\frac{\bar{\rho} \widetilde{w}}{\widetilde{T}} & 0 & 0 & \widetilde{w} \frac{\partial \bar{\rho}}{\partial \widetilde{Y}_{1}} & \ldots & \widetilde{w} \frac{\partial \bar{\rho}}{\partial \widetilde{Y}_{N_{k}-1}} \\
\frac{\widetilde{\rho}}{\beta} & 0 & 0 & 0 & -\frac{\bar{\rho} q}{\widetilde{T}} & \bar{\rho} & 0 & q \frac{\partial \bar{\rho}}{\partial \widetilde{Y}_{1}} & \ldots & q \frac{\partial \bar{\rho}}{\partial \widetilde{Y}_{N_{k}-1}} \\
\frac{\omega}{\beta} & 0 & 0 & 0 & -\frac{\bar{\rho} \omega}{\widetilde{T}} & 0 & \bar{\rho} & \omega \frac{\partial \bar{\rho}}{\partial \widetilde{Y}_{1}} & \ldots & \omega \frac{\partial \bar{\rho}}{\partial \widetilde{\rho}_{N_{k}-1}} \\
\frac{\widetilde{\varphi}}{\beta} & 0 & 0 & 0 & -\frac{\bar{\rho} \widetilde{Y_{1}}}{\widetilde{T}} & 0 & 0 & \bar{\rho}+\widetilde{Y}_{1} \frac{\partial \bar{\rho}}{\partial \widetilde{Y}_{1}} & \ldots & \widetilde{Y}_{1} \frac{\partial \bar{\rho}}{\partial \widetilde{Y}_{N_{k}-1}} \\
\vdots & \vdots & \vdots & \vdots & \vdots & \vdots & \vdots & \vdots & \ddots & \vdots \\
\widetilde{Y}_{N_{k}-1} & 0 & 0 & 0 & -\frac{\bar{\rho} \widetilde{Y}_{N_{k}-1}}{\widetilde{T}} & 0 & 0 & \widetilde{Y}_{N_{k}-1} \frac{\partial \bar{\rho}}{\partial \widetilde{Y}_{1}} & \ldots & \bar{\rho}+\widetilde{Y}_{N_{k}-1} \frac{\partial \bar{\rho}}{\partial \widetilde{Y}_{N_{k}-1}}
\end{array}\right]
$$

with the preconditioning function $\beta$ defined as

$$
\beta=\left\{\begin{array}{lll}
\widetilde{\mathbf{U}}^{2} / \gamma & & \varepsilon<M a<1, \\
R \widetilde{T} & \text { for } & M a \geq 1, \\
\varepsilon^{2} c^{2} / \gamma & & M a \leq \varepsilon,
\end{array}\right.
$$

using the velocity vector $\widetilde{\mathbf{U}}$ and the isentropic exponent $\gamma$. The parameter $\varepsilon$ is a small number $(\ll 1)$ which ensures stability in terms of singularity prevention close to stagnation points. In the preconditioned system the eigenvalues in $x$-direction for $M a \rightarrow 0$ approach $\lambda_{x, 1,2}=\widetilde{u}(1 \pm \sqrt{5}) / 2$, and the multiple eigenvalue $\widetilde{u}$. Accordingly, the condition number reaches a finite value.

$\boldsymbol{\Gamma}$ originates from the preconditioner of Choi and Merkle [33]. However the derivatives of density with respect to temperature are not neglected and the definition of $\beta$ is a continuous function which is chosen so that the preconditioning matrix $\boldsymbol{\Gamma}$ reduces to the transformation matrix $\partial \mathbf{Q}_{c} / \partial \mathbf{Q}_{p}$ for 
$M a \geq 1$. Consequently, the original set of equations (1) is recovered and solved in the supersonic flow regime.

\subsection{High order interface value reconstruction by $M L P^{l d}$}

For the discretization of the inviscid fluxes at a cell interface the $\mathrm{AUSM}^{+}$-up flux vector splitting [29] is employed. It requires left and right variable vectors at any cell interface. They are calculated by a high order (up to sixth order) polynomial approach which usually causes stability problems. Therefore the multi-dimensional limiting process MLP [20, 1] is used, in the version of [2]. MLP enables convergence in cases where standard TVD schemes fail. It interacts with the TVD limiter in such a way that local extrema at the corner points of a volume are avoided. The discretization of the inviscid fluxes is not done in each coordinate direction separately. Instead MLP uses information from diagonal volumes and thus combines the different coordinate directions. This stabilizes the numerical scheme and allows better results if shock waves are oblique to the computational grid $[20,1,2]$. Because MLP is a very new technique, the most important details are explained in this section.

From the cell center averages at $i-2, i-1, \ldots, i+3$ of a variable $q$, the unlimited left $(L)$ and right $(R)$ interface states at $i+1 / 2$ are calculated by

$$
\begin{aligned}
& q_{i+1 / 2}^{L, x}=q_{i}+0.5 \beta_{i}^{L, x}(r) \Delta q_{i-1 / 2}, \\
& q_{i+1 / 2}^{R, x}=q_{i+1}-0.5 \beta_{i+1}^{R, x}(r) \Delta q_{i+3 / 2},
\end{aligned}
$$

where $\beta^{x}(r)$ is a function which defines order and type of discretization. It depends on a number of slope ratios $r_{i}^{L}=\Delta q_{i+1 / 2} / \Delta q_{i-1 / 2}$ and $r_{i}^{R}=\Delta q_{i-1 / 2} / \Delta q_{i+1 / 2}$ which are calculated by $\Delta q_{i+1 / 2}=q_{i+1}-q_{i}$. The parameters $\beta^{x}(r)$ used in this paper are summarized in Table I. The second order discretization $(x=2 O)$ corresponds to the van Leer limiter. The remaining discretizations ( $x=3 O, 4 O, 5 O$, third to fifth order) are unlimited upwind biased stencils. By 
Table I. Functions $\beta_{i+1 / 2}^{L, x}$ and $\beta_{i+1 / 2}^{R, x}$ for a second order van Leer $(x=2 O)$ and third to fifth order upwind biased $(x=3 O$ to $5 O)$ discretizations.

\begin{tabular}{ccc}
\hline$x$ & $\beta_{i+1 / 2}^{L, x}$ & $\beta_{i+1 / 2}^{R, x}$ \\
\hline $2 O$ & $2 r_{i}^{L} /\left(1+r_{i}^{L}\right)$ & $2 r_{i+1}^{R} /\left(1+r_{i+1}^{R}\right)$ \\
$3 O$ & $\left(1+2 r_{i}^{L}\right) / 3$ & $\left(1+2 r_{i+1}^{R}\right) / 3$ \\
$4 O$ & $\left(-1 / r_{i-1}^{L}+4+3 r_{i}^{L}\right) / 6$ & $\left(3 r_{i+1}^{R}+4-1 / r_{i+2}^{R}\right) / 6$ \\
$5 O$ & $\left(-2 / r_{i-1}^{L}+11+24 r_{i}^{L}-3 r_{i}^{L} r_{i+1}^{L}\right) / 30$ & $\left(-3 r_{i}^{R} r_{i+1}^{R}+24 r_{i+1}^{R}+11-2 / r_{i+2}^{R}\right) / 30$ \\
\hline
\end{tabular}

averaging the left and right fifth order values

$$
q_{i+1 / 2}^{6 O}=q_{i+1 / 2}^{L, 6 O}=q_{i+1 / 2}^{R, 6 O}=0.5\left(q_{i+1 / 2}^{L, 5 O}+q_{i+1 / 2}^{R, 5 O}\right)
$$

a central sixth order discretization is obtained. Because the polynomial reconstruction (7) is not able to achieve well resolved, non-oscillatory solutions at discontinuities Kim et al. [20] intoduced a filtering of the unlimited values using the MLP parameter $1 \leq \alpha \leq 2$

$$
\begin{aligned}
q_{i+1 / 2}^{L} & =q_{i}+0.5 \max \left[0, \min \left(\alpha^{L}, \alpha^{L} r_{i}^{L}, \beta_{i}^{L}\right)\right] \Delta q_{i-1 / 2}, \\
q_{i+1 / 2}^{R} & =q_{i+1}-0.5 \max \left[0, \min \left(\alpha^{R}, \alpha^{R} r_{i+1}^{R}, \beta_{i+1}^{R}\right)\right] \Delta q_{i+3 / 2} .
\end{aligned}
$$

For $\alpha=2$ this limiation becomes identical to the TVD constraint of Sweby [34]. The MLP domain is a subset of the TVD region. In Eq. (9) any higher order discretizations may be used by an appropriate choice of $\beta(r)$. Standard limiters (e.g. the van Leer limiter) which are automatically is in the second order TVD region may be additionally limited by MLP. The parameter $\alpha$ performs a linear scaling from the upper TVD limit $(\alpha=2)$ defined by Sweby to the more viscous lower limit $(\alpha=1)$ which corresponds to the minmod limiter. The new parameter $\alpha$ is introduced to avoid local extrema at the corner points of a computational volume. If there is no local extrema $\alpha=2$ is chosen and any high order discretization is limited by the upper TVD constraint only. For practical reasons $\alpha \in[0,2]$ may be also used without adding too much numerical dissipation [2]. It has been shown, that the additional MPL limitation improves the results and convergence properties $[20,1,2]$. However, as for any TVD limiter the scheme switches to first order if there is a local extremum in 
the corresponding coordinate direction. This may cause too much numerical dissipation e.g. in a LES (large eddy simulation). On the other hand, the unlimited sixth order central discretization according to Eq. (8) is dispersive and does not offer enough numerical dissipation to be used in practical applications. For this reason the following blend is used by the authors for LES [35] and also for one of the test cases shown in this paper

$$
\begin{aligned}
& q_{i+1 / 2}^{L, 5 / 6 O}=q_{i+1 / 2}^{6 O}+S\left(q_{i+1 / 2}^{L, 5 O}-q_{i+1 / 2}^{6 O}\right) \\
& q_{i+1 / 2}^{R, 5 / 6 O}=q_{i+1 / 2}^{6 O}+S\left(q_{i+1 / 2}^{R, 5 O}-q_{i+1 / 2}^{6 O}\right)
\end{aligned}
$$

with $S \leq 0.15$. For $S=0.1$ this corresponds to $90 \%$ sixth order unlimited central discretization and $10 \%$ fifth order upwind biased MLP/TVD discretization. For unsteady simulations this has proven to be a good choice.

2.2.1. Calculation of $\alpha$ and final $M L P^{l d}$ scheme: The basic point of MLP is the calculation of $\alpha$ which is shortly explained now. For details and proofs concerning the MLP ${ }^{l d}$ version used in this paper see Ref. [2]. The idea behind MLP is that corner values are calculated for each volume from the cell interface values. These corner values are not allowed to be a local extremum. This is achieved by an appropriate choice of the parameter $\alpha$. If $\alpha=1$ is used in Eq. (9) the part involving the $\alpha$ values define a lower MLP limit wich corresponds to the symmetric minmod $(\mathrm{mm})$ limiter

$$
\Delta q^{m m}=2\left(q_{i+1 / 2}^{L, m m}-q_{i}\right)=2\left(q_{i}-q_{i-1 / 2}^{R, m m}\right)=\max \left[0, \min \left(1, r_{i}\right)\right] \Delta q_{i-1 / 2} .
$$

For $\alpha<1$ the scheme is not longer second order. On the other hand $\alpha \leq 2$ is needed to achieve TVD properties. Using $\Delta q^{m m}$ and $\alpha$ as a parameter which still has to be determined, the eight (in 3D) corner value $q^{M L P}$ of a volume of a structured grid are calculated with $\kappa_{x}, \kappa_{y}, \kappa_{z}= \pm 1$ by

$$
q_{i+\kappa_{x} / 2, j+\kappa_{y} / 2, k+\kappa_{z} / 2}^{M L P}=q_{i, j, k}+\frac{1}{2} \kappa_{x} \alpha_{x} \Delta q_{x}^{m m}+\frac{1}{2} \kappa_{y} \alpha_{y} \Delta q_{y}^{m m}+\frac{1}{2} \kappa_{z} \alpha_{z} \Delta q_{z}^{m m},
$$


in the $x-, y-$, and $z$-direction. These corner values $q^{M L P}$ are now limited according to

$$
Q_{i+\kappa_{x} / 2, j+\kappa_{y} / 2, k+\kappa_{z} / 2}^{\min } \leq q_{i+\kappa_{x} / 2, j+\kappa_{y} / 2, k+\kappa_{z} / 2}^{M L P} \leq Q_{i+\kappa_{x} / 2, j+\kappa_{y} / 2, k+\kappa_{z} / 2}^{\max }
$$

by an appropriate choice of $\alpha_{i}$. The lower and upper limits $Q^{\min }$ and $Q^{\max }$ are defined to be the minimum or maximum value out of the eight cell center values surrounding one corner [20]. From the eight corner values of a volume only two have to be checked for a maximum $\left(Q_{1}^{\max }\right.$ and $\left.Q_{2}^{\max }\right)$ and a minimum $\left(Q_{1}^{\min }\right.$ and $\left.Q_{2}^{\min }\right)$ [2]. The first corner to be checked for a maximum is $\mathbf{C}_{1}^{\max }$ located at imax,jmax, kmax and for a minimum $\mathbf{C}_{1}^{\min }$ located at imin,jmin,kmin. The corner indices are obtained from

\begin{tabular}{|c|c|c|c|c|c|c|c|c|}
\hline & imax & imin & & $j \max$ & jmin & & $k \max$ & kmin \\
\hline$\Delta q_{x}^{m m}>0$ & $i+\frac{1}{2}$ & $i-\frac{1}{2}$ & $\Delta q_{y}^{m m}>0$ & $j+\frac{1}{2}$ & $j-\frac{1}{2}$ & $\Delta q_{z}^{m m}>0$ & $k+\frac{1}{2}$ & $k-\frac{1}{2}$ \\
\hline$\Delta q_{x}^{m m}<0$ & $i-\frac{1}{2}$ & $i+\frac{1}{2}$ & $\Delta q_{y}^{m m}<0$ & $j-\frac{1}{2}$ & $j+\frac{1}{2}$ & $\Delta q_{z}^{m m}<0$ & $k-\frac{1}{2}$ & $k+\frac{1}{2}$ \\
\hline
\end{tabular}

The indices of the second point to be checked for a maximum $\left(\mathbf{C}_{2}^{\max }\right)$ and for a minimum $\left(\mathbf{C}_{2}^{\min }\right)$, respectively, follow from

\begin{tabular}{ccc} 
& $\mathbf{C}_{2}^{\max }$ & $\mathbf{C}_{2}^{\min }$ \\
\hline$\left|\Delta q_{x}^{m m}\right|<\min \left(\left|\Delta q_{y}^{m m}\right|,\left|\Delta q_{z}^{m m}\right|\right)$ & imin,jmax,kmax & imax,jmin,kmin \\
$\left|\Delta q_{y}^{m m}\right|<\min \left(\left|\Delta q_{x}^{m m}\right|,\left|\Delta q_{z}^{m m}\right|\right)$ & imax,jmin,kmax & imin,jmax, kmin \\
$\left|\Delta q_{z}^{m m}\right|<\min \left(\left|\Delta q_{x}^{m m}\right|,\left|\Delta q_{y}^{m m}\right|\right)$ & imax,jmax,kmin & imin,jmin, kmax
\end{tabular}.

The check of both corners may be combined in one condition [2] defining the maximum absolute change $\Delta q^{c}$ which still fulfills Eq. (13)

$$
\begin{array}{r}
\Delta q^{c}=2 \min \left(Q_{1}^{\max }-q_{i, j, k}, Q_{2}^{\max }-q_{i, j, k}+2\left|\Delta q_{\min }^{m m}\right|,\right. \\
\left.q_{i, j, k}-Q_{1}^{\min }, q_{i, j, k}-Q_{2}^{\min }+2\left|\Delta q_{\min }^{m m}\right|\right) .
\end{array}
$$


Here $\left|\Delta q_{\min }^{m m}\right|=\min \left(\left|\Delta q_{x}^{m m}\right|,\left|\Delta q_{y}^{m m}\right|,\left|\Delta q_{z}^{m m}\right|\right)$ is the smallest absolute change of $\Delta q^{m m}$ from the three coordinate directions.

While in the one-dimensional case there is a unique value of $\alpha$ to fulfill Eq. (13), this is not the case for two- or three-dimensional flows. As outlined in [2], in the MLP ${ }^{l d}$ version used in this paper $\alpha_{x}, \alpha_{y}, \alpha_{z}$ are chosen in order

1. to fulfill Eq. (13) which is achieved by

$$
\left|\Delta q^{M L P}\right|=\alpha_{x}\left|\Delta q_{x}^{m m}\right|+\alpha_{y}\left|\Delta q_{y}^{m m}\right|+\alpha_{z}\left|\Delta q_{z}^{m m}\right|=\Delta q^{c}
$$

Note that the $\leq$ sign from Eq. (13) is replaced by an equality sign to minimize the impact from MLP,

2. to keep the change in mean gradient direction (calculated with central discretization)

$$
\left|\Delta \bar{q}_{x}\right|=\left|q_{i+1, j, k}-q_{i-1, j, k}\right|, \quad\left|\Delta \bar{q}_{y}\right|=\left|q_{i, j+1, k}-q_{i, j-1, k}\right|, \quad\left|\Delta \bar{q}_{z}\right|=\left|q_{i, j, k+1}-q_{i, j, k-1}\right|
$$

by the introduction of $\alpha_{i}$ as small as possible.

$\left|\Delta q^{M L P}\right|=\Delta q^{c}=$ constant defines a limiting plane in the three-dimensional discretization space $\left|\Delta q_{x}\right|,\left|\Delta q_{y}\right|,\left|\Delta q_{z}\right|$ [2]. Additional limitations are given by the TVD constraint. Any point on this plane fulfills Eq. (16). To keep the second condition given above, the coordinates of point $\mathbf{A}\left(\mathbf{A}_{\mathbf{x}}, \mathbf{A}_{\mathbf{y}}, \mathbf{A}_{\mathbf{z}}\right)$ representing the intersection between the line of the absolute mean gradient direction and the limiting plane $\left|\Delta q^{M L P}\right|=\Delta q^{c}=$ constant is calculated by

$$
A_{x}=f\left|\Delta \bar{q}_{x}\right|, \quad A_{y}=f\left|\Delta \bar{q}_{y}\right|, \quad A_{z}=f\left|\Delta \bar{q}_{z}\right|
$$

using the scaling factor

$$
f=\Delta q^{c} /\left(\left|\Delta \bar{q}_{x}\right|+\left|\Delta \bar{q}_{y}\right|+\left|\Delta \bar{q}_{z}\right|\right) .
$$


Aim is to obtain a solution which agrees as much as possible with the mean gradient direction [2]. If point $\mathbf{A}$ is located inside the TVD region it directely defines the final $\alpha$ values. However, if $A$ is outside the TVD region it has to be moved on the plane $\left|\Delta q^{M L P}\right|=\Delta q^{c}=$ constant until the required conditions are met. Using the results from Eqs. (11) to (19) the corresponding procedure to calculate $\alpha_{x}, \alpha_{y}, \alpha_{z} \in[0,2]$ is:

$$
\operatorname{MLP}^{l d}\left\{\begin{aligned}
& \text { set } \alpha_{i}=2 \\
&\text { if } \left.\left(2\left(\left|\Delta q_{x}^{m m}\right|+\left|\Delta q_{y}^{m m}\right|\right)+\left|\Delta q_{z}^{m m}\right|\right)>\Delta q^{c}\right) \text { then } \\
& \text { do for } i=x, y, z \\
& h_{i}=\max \left(A_{i}-2\left|\Delta q_{i}^{m m}\right|, 0\right) \\
& U_{i}=A_{i}-h_{i}, \quad U_{j}=A_{j}+h_{i} / 2 \text { for } j=x, y, z \text { and } j \neq i \\
& f=h_{i} /\left(h_{i}+\epsilon\right) \quad \text { with } \epsilon \text { is a small number } \\
& l= f\left[\max \left(A_{j}-2\left|\Delta q_{j}^{m m}\right|, 0\right)-\max \left(A_{k}-2\left|\Delta q_{k}^{m m}\right|, 0\right)\right] \\
& j, k=x, y, z \text { and } j, k \neq i \text { and } j \neq k \\
& S_{i}=U_{i}, \quad S_{j}=U_{j}-l, \quad S_{k}=U_{k}+l \\
& \alpha_{n}=S_{n} /\left|\Delta q_{n}^{m m}\right|, \quad A_{n}=S_{n} \quad \text { for } n=x, y, z \\
& \text { end do } \\
& \text { end if } \quad
\end{aligned}\right.
$$

The calculation of the final values $\alpha_{i}$ requires a loop over $x, y$, and $z$. In some cases the final values are already obtained after the first iteration and remain unchanged in the following ones.These $\alpha_{x}$, $\alpha_{y}$, and $\alpha_{z}$ values can be used in Eq. (9) in combination with any higher order approach for $\beta$.

\section{NUMERICAL SOLVER FOR THE SOLID}

HeatEQ [23] applies central differences for a second order accurate spatial discretization. To integrate the three-dimensional heat equations in time the Douglas-Gunn Alternating Direction Implicit (ADI) method [36] is used. It is a three step procedure, which is unconditionally stable 
and second order accurate in time. The tridiagonal system of linear equations resulting from each ADI step is efficiently solved by a Thomas algorithm [37, 38]. The code HeatEQ is fully vectorized.

\subsection{Coupling of the simulations}

After a certain number of iterations converged solutions for fixed wall temperature conditions are reached in the internal and external gas phase flow simulations. Next the data required for conjugate heat transfer $\left(\lambda_{g}, T_{g}, \Delta y_{g}\right)$ is interpolated linearly to the adjacent mesh of the solid injector (see explanation in section 5.3). These values are submitted to the heat conduction solver for the solid. In return the solid phase solver communicates the resulting steady-state wall temperatures (by interpolation to the corresponding near wall grids) to the internal and external gas phase flow simulations. The coupling of the gas phase flows with the heat conduction in the solid is continued until an overall steady-state is reached (more details are given in section 5.4)

\section{CODE VALIDATION}

\subsection{Gas phase flow}

TASCOM3D has been validated for supersonic reacting [39, 12, 40] and non-reacting [21, 28, $12,22,2]$ flows using a large number of experimental test cases. In order to validate the newly implemented preconditioning technique and the high order discretization several subsonic test cases have been simulated. For the sake of brevity two of them are presented in the following paragraphs only:

1. the flows in convergent-divergent nozzles covering a wide range of sub- and supersonic Mach numbers and

2. the flow in a lid driven cavity that is characterized by particularly small flow velocities.

The first test case is chosen to demonstrate the improved convergence behavior in case of preconditioning and the second one to show the ability of the high order discretization to achieve 
Table II. Geometry of the test case, number of volumes, entry, and exit Mach numbers for three convergentdivergent nozzles with given area ratios (ration of inlet area to area at the nozzle throat).

\begin{tabular}{cccc}
\hline$A_{\text {in }} / A^{*}$ & 10 & 100 & 1340 \\
\hline geometry & planar & axisymmetric & axisymmetric \\
grid cells & $140 \times 30$ & $140 \times 30$ & $140 \times 60$ \\
$M a_{\text {in }}$ & $5.7 \cdot 10^{-2}$ & $5.8 \cdot 10^{-3}$ & $4.5 \cdot 10^{-4}$ \\
$M a_{\text {out }}$ & 2.18 & 2.87 & 3.61 \\
\hline
\end{tabular}

excellent results at low additional numerical cost. Moreover, the simulation of the second test case only became possible by using the preconditioning technique.

4.1.1. Convergent-divergent nozzles One planar and two rotationally symmetric nozzles are simulated with and without the preconditioned technique. The area ratios $A_{\text {in }} / A^{*}\left(A_{\text {in }}\right.$ is the area at the inlet, $A^{*}$ at the nozzle throat) as well as the Mach numbers at their entries and at the exits are listed in Tab. II. After the flow enters the nozzles with low subsonic speed, it is accelerated in the convergent parts up to Mach one in the nozzle throats, and is further expanded to supersonic speeds in the diverging parts of the nozzles. The computational grids are refined in the near-wall regions.

In the results of these simulations no differences arise from preconditioning for the area ratios $A_{\text {in }} / A^{*}=10$ and 100 . For $A_{\text {in }} / A^{*}=1340$ minor differences are observed close to the nozzle entry in the very low Mach number region $(M a<0,001)$. These very small deviations of the isocontours of Mach number might be first signs of an inaccurate solution of the non-preconditioned simulation. Moreover the convergence rates are significantly improved by the preconditioning as may be seen from Fig. 1 where the averaged density residuals of simulations with and without preconditioning are compared.

4.1.2. Lid driven cavity flow The lid driven cavity flow is a standard test case for the validation of incompressible flow solvers. It considers the two-dimensional laminar flow in a square cavity bounded by isothermal no-slip walls $\left(T_{\text {wall }}=293 \mathrm{~K}\right)$. The flow is driven by the top wall of the cavity, which is moving at a constant velocity in positive $x$-direction. Depending on the Reynolds number, which is based on the length of the cavity and the lid velocity, characteristic vortices and 

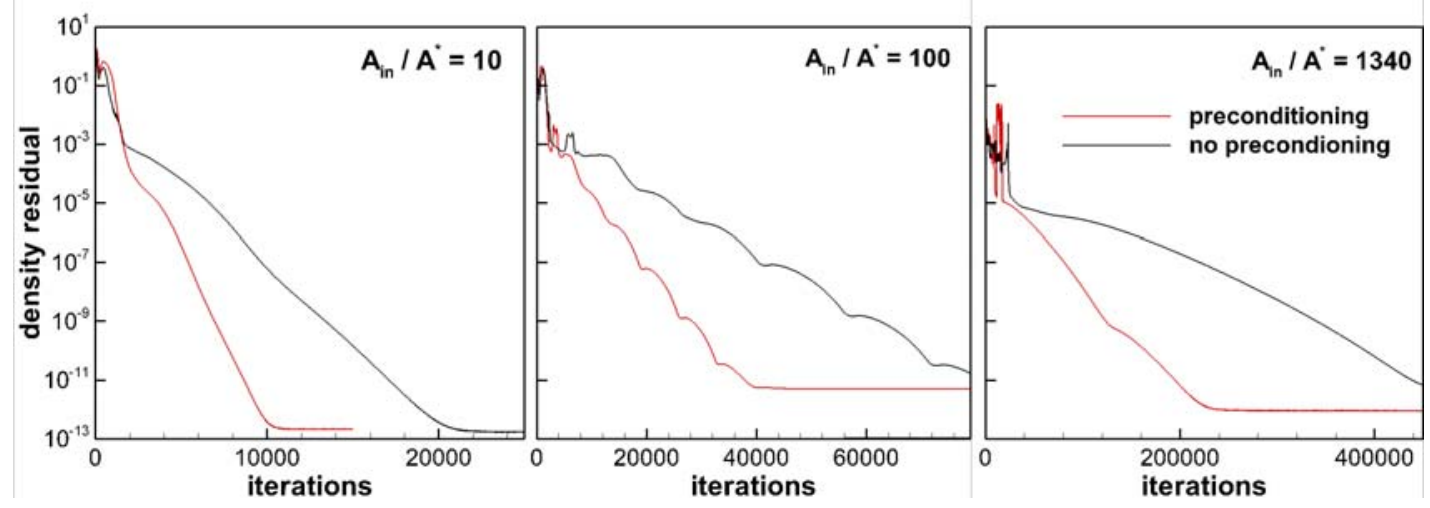

Figure 1. Convergence histories with (solid line) and without (dashed line) preconditioning for convergentdivergent nozzles with different area ratios.
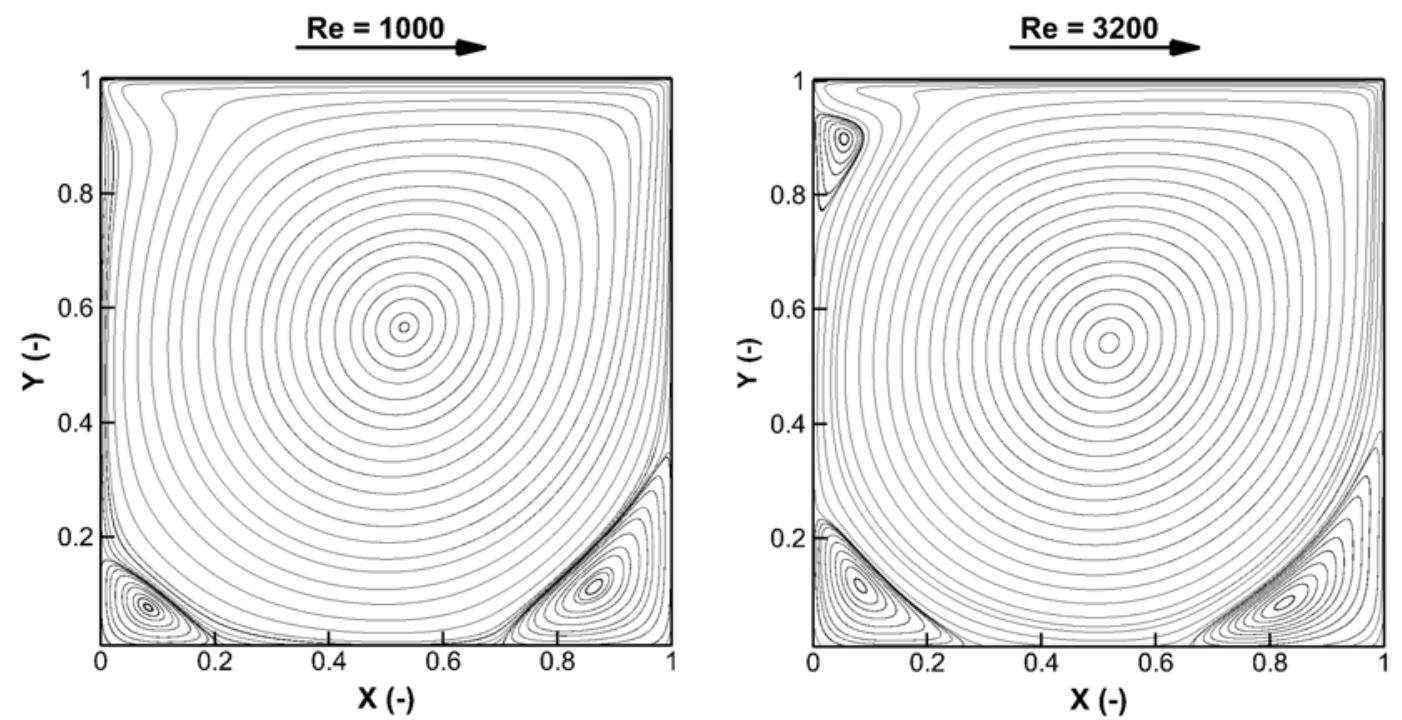

Figure 2. Streamlines in the cavity for $R e=1000$ (left) and $R e=3200$ (right).

flow patterns arise. Two different Reynolds numbers are investigated in this paper: $R e=1000$ and $R e=3200$. Due to very low Mach numbers smaller than $M a_{\max }=0.0003$ for $R e=1000$ and $M a_{\max }=0.00096$ for $R e=3200$ solving the original set of equations was not possible and preconditioning is required. As this is a laminar test case no turbulence model is applied. An equidistant mesh with $200 \times 200$ cells is used. Best results are obtained using a blend of $95 \%$ sixth order central and $5 \%$ fifth order upwind $\mathrm{MLP}^{l d}$ discretization (to add some numerical dissipation) according to Eq. (10) with $S=0.05$. 
Streamlines of the converged solutions are shown in Fig. 2 for $R e=1000$ (left side) and $R e=3200$ (right side). Normalized velocities in $x$ - and $y$-direction along the vertical and horizontal lines through the center of the cavity are plotted in Fig. 3 together with experimental data of Ghia et al. [41]. They are in excellent agreement. Results are plotted for both investigated Reynolds numbers. The benefit from using a high order discretization may be seen from Fig. 4, where velocity profiles are compared for different orders of discretization ( $R e=1000$ case). While in many turbulent steady-state simulations the differences between second and higher order discretizations are relatively small [2], this laminar steady-state test case shows a strong influence with respect to the chosen type of discretization. Figure 4 shows large discrepancies in comparison to the data of Ghia et al. for the simulation with second order discretization using the van Leer limiter. However, the plots progressively approach the experimental data when the order of discretization is increased.

The poor results of the second order scheme can be improved by grid refinement (see Fig. 5).
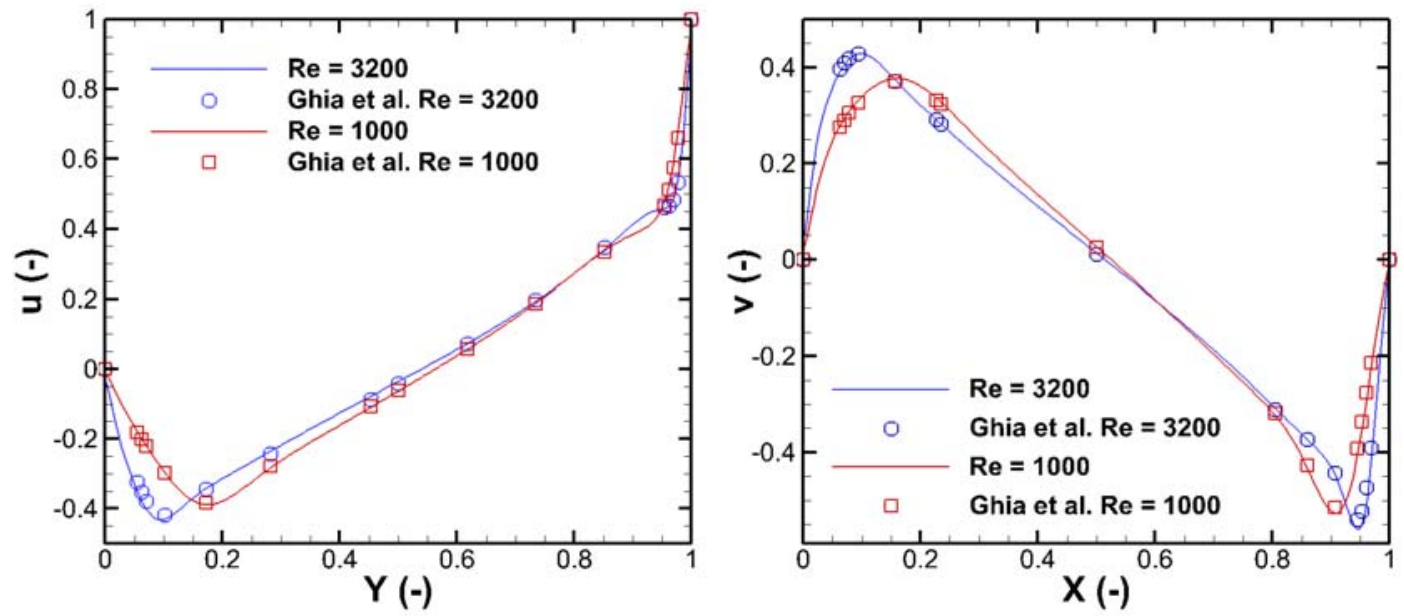

Figure 3. Normalized velocity in $x$-direction along the vertical line through the center of the cavity (left) and in $y$-direction along the horizontal line through the center of the cavity (right) using a 5th/6th order spatial discretization.

But even if the number of equidistant cells in each direction is quadrupled ( $800 \times 800$ volumes), it does not achieve the same level of agreement as the combined fifth/sixth order discretization on the $200 \times 200$ grid. 

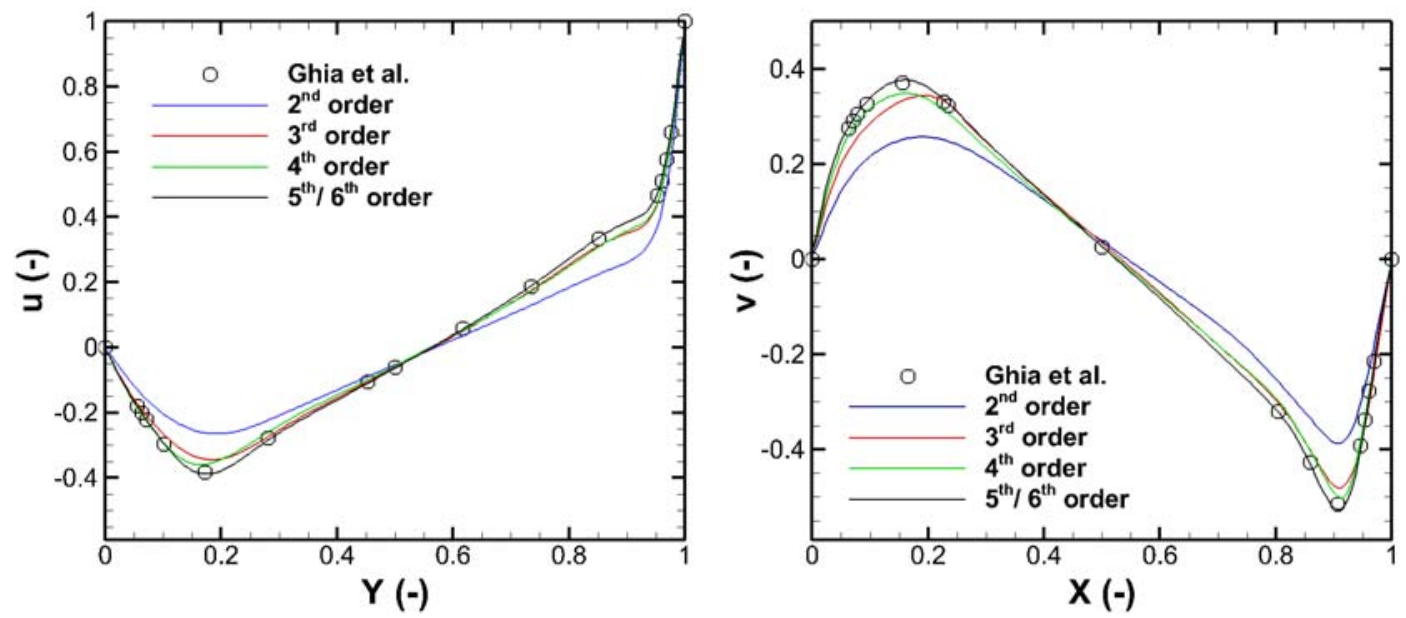

Figure 4. Normalized velocity in $x$-direction along the vertical line through the center of the cavity (left) and in $y$-direction along the horizontal line through the center of the cavity (right) for different orders of spatial discretization $(R e=1000)$.
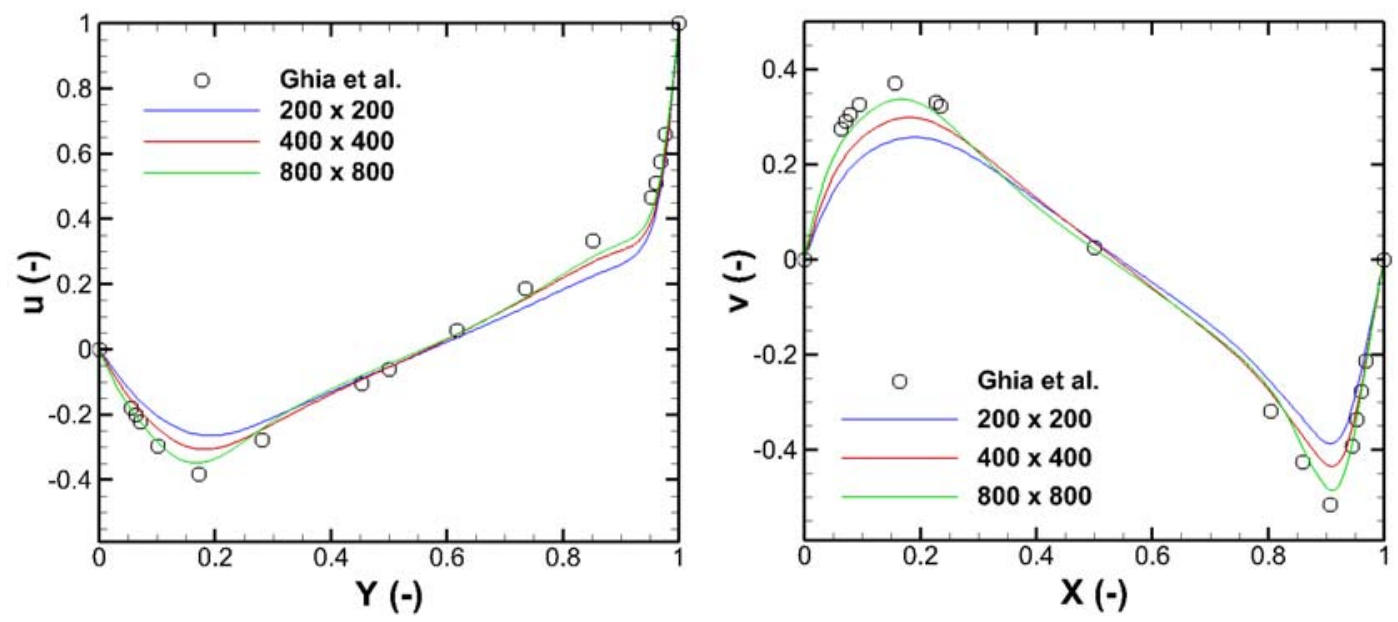

Figure 5. Normalized velocity in $x$-direction along the vertical line through the center of the cavity (left) and in $y$-direction along the horizontal line through the center of the cavity (right) for three different equidistant grids $(R e=1000)$ using the 2 nd order van Leer limiter.

\subsection{Heat Conduction in a solid}

The in-house code HeatEQ has been validated [23] using several test cases with available theoretical or experimental results. HeatEQ shows excellent agreement with the analytical solution for the temporal evolution of the temperature distribution in an infinite flat plate with a constant heat transfer coefficient at its upper and lower boundaries for a given ambient temperature. The analytically found temperature distribution for a finite flat plate with constant temperatures at its 


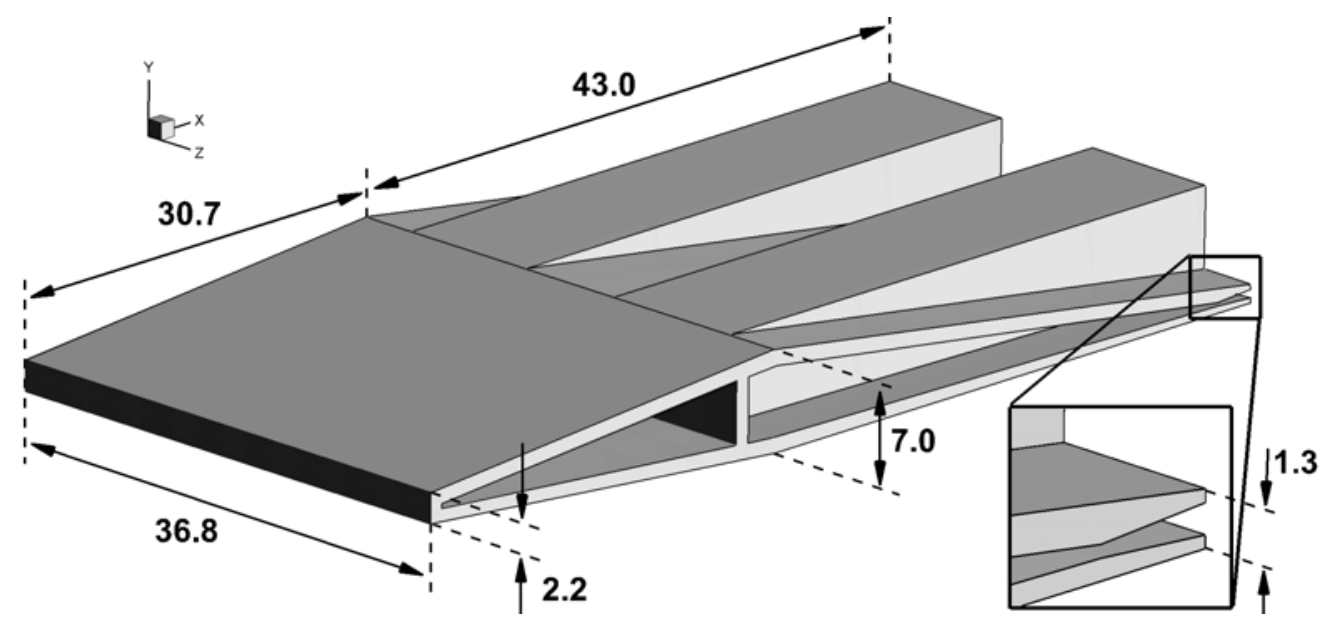

Figure 6. Injector geometry and enlarged view of the nozzle inside the strut (dimensions in mm).

boundaries has also been reproduced successfully. Moreover, conjugate heat transfer has been tested for a supersonic Mach 2.6 boundary layer over a flat plate.

\section{SCRAMJET STRUT INJECTOR SIMULATION}

The strut injector studied in this paper uses a lobed structure for the creation of streamwise vorticity to achieve an enhanced mixing of fuel and air. It has been investigated experimentally [12, 42, 19] at the Institute of Aerospace Thermodynamics at the University of Stuttgart. Numerical studies have been performed to analyze variations of the strut geometry [43, 44, 14] and fuel injection areas $[45,13]$ with regard to the mixing and combustion performances. In addition to these more fundamental studies which have been conducted for model combustors under laboratory conditions, the lobed strut injector has also been investigated in a complete scramjet demonstrator model at real flight conditions [11].

\subsection{Geometry of the strut injector and combustor section}

The geometry used for the heat transfer and cooling investigation is shown in Fig. 6. In contrast to previously performed studies, the sharp tip at the leading edge of the strut is removed, because its slim shape is not suitable for an efficient active cooling. Thereby the original strut length of $86 \mathrm{~mm}$ 
is reduced to $73.7 \mathrm{~mm}$ and a small bow shock is generated resulting in a small subsonic region in front of the blunt leading edge of the strut.

The strut injector is mounted centrally from one side wall to the other in a channel of constant cross section. Figure ?? shows the investigated section of the channel. In the strut injector studies mentioned before detached flames have been observed which stabilize downstream of the strut. Accordingly, the thermal effect on the strut injector due to combustion is assumed to be minor. Moreover, for a practical use it is essential, that the flame does not extend upstream of the strut's tail because this can cause thermal choking. For these reasons, the main focus is on the heat transfer from the extremely hot external outer gas through the strut towards the cool interior hydrogen and combustion is not considered. Consequently, the nearfield of the strut - starting $32.3 \mathrm{~mm}$ upstream of its leading edge and ending $50 \mathrm{~mm}$ downstream of its trailing edge - is simulated only. In order to reduce the computational cost one half of the symmetrical channel is selected as computational domain (highlighted in Fig. ??). At the rear of the injector (at $x=86 \mathrm{~mm}$ ) gaseous hydrogen is injected in axial flow direction. The trailing edge of the strut injector as well as the used horizontal injection ports are shown in Fig. 8. To achieve an efficient cooling a relatively complex internal

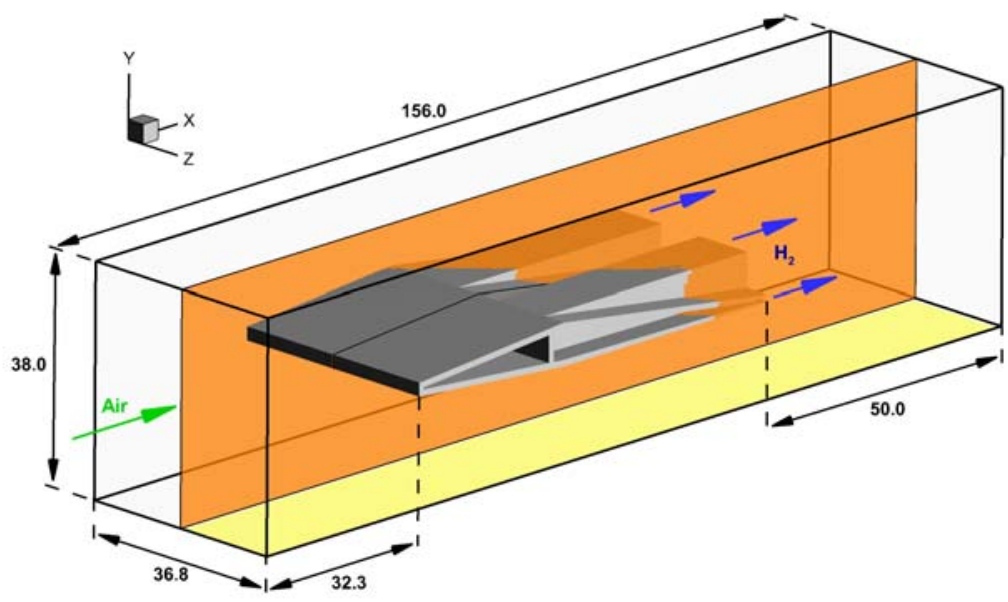

Figure 7. Trailing edge of the lobed strut injector (dimensions in $\mathrm{mm}$ ).

structure for the strut is required which is depicted in Fig. 9. Cold, gaseous hydrogen enters the 


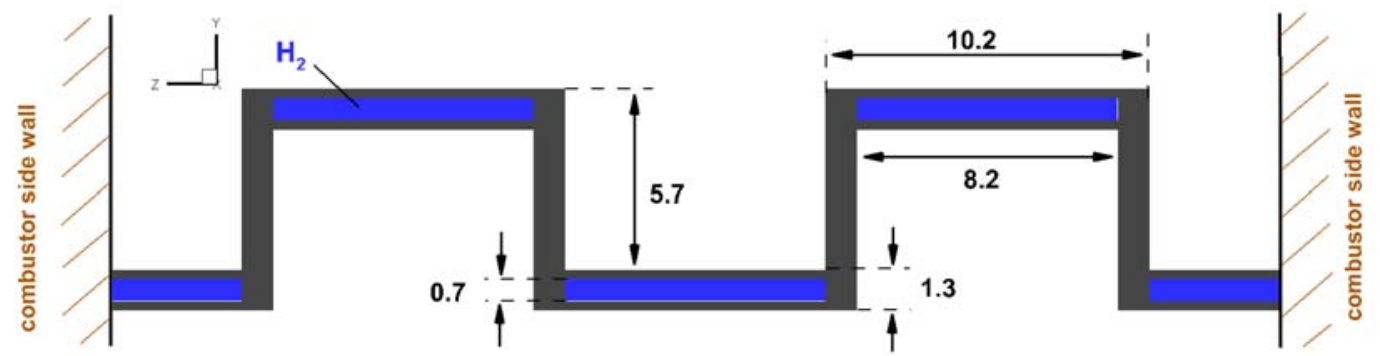

Figure 8. Trailing edge of the lobed strut injector (dimensions in $\mathrm{mm}$ ).

injector via two channels through the combustor side walls. Guided by internal walls the hydrogen is first directed towards the strut tip, where the highest thermal loads are expected. Then the flow turns back and the hydrogen is distributed over the different ramps of the injector towards the exit. At the end of the ramps convergent-divergent nozzles accelerate the flow to supersonic speed (see enlarged view in Fig. 6). The wall thickness at the leading edge is $0.9 \mathrm{~mm}$. The other internal and external injector walls have a thickness of $1 \mathrm{~mm}$ except for the nozzle regions at the end of the ramps where the walls are thinner. 


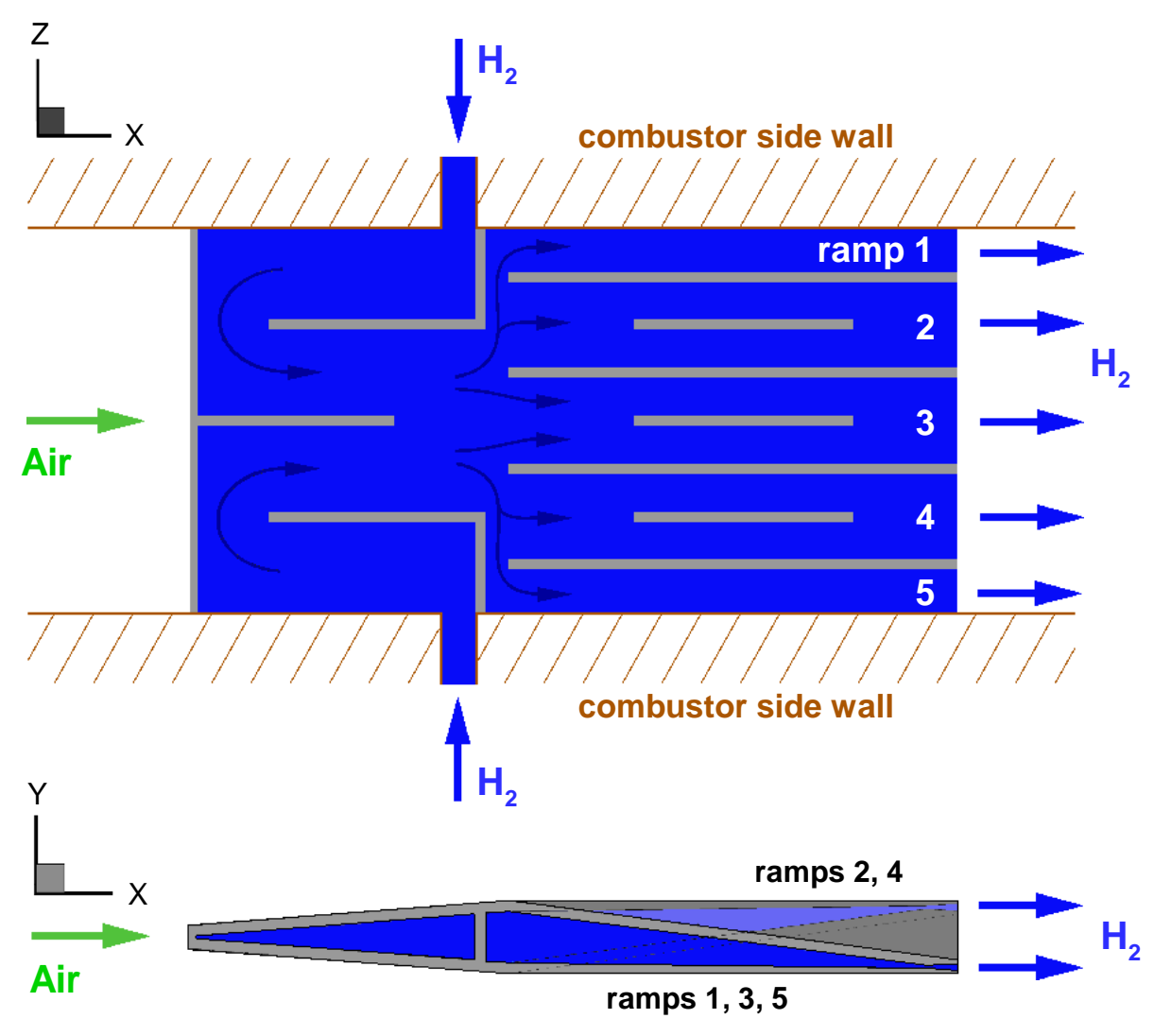

Figure 9. Internal geometry of the strut (top: top view of the midplane; bottom: side view).

\subsection{Boundary conditions}

The air inflow conditions at the combustion chamber entrance correspond to a scramjet flight Mach number of approximately 8 . Table III lists the combustor inflow values as well as the hydrogen inflow conditions for the supply channels in the combustor side walls. The resulting equivalence ratio is $\Phi=0.72$. For the combustor side walls and the hydrogen supply channels a wall temperature of $T_{w}=450 \mathrm{~K}$ is assumed. The same wall temperature is used for the strut injector to initialize the simulation. The simulations for the strut external and internal flows use the fourth order MLP ${ }^{l d}$ discretization. 
Table III. Inflow conditions for air (main flow) and hydrogen (at the supply tubes)

\begin{tabular}{lcc}
\hline & air & $\mathbf{H}_{\mathbf{2}}$ \\
\hline Mach number $M a(-)$ & 3.0 & 0.13 \\
static pressure $p($ bar $)$ & 0.97 & 44.5 \\
static temperature $T(\mathrm{~K})$ & 1160 & 290 \\
total temperature $T_{t}(\mathrm{~K})$ & 3250 & 291 \\
velocity $u_{\infty}(\mathrm{m} / \mathrm{s})$ & 2003 & 170 \\
\hline
\end{tabular}

\subsection{Computational grids}

Table IV summarizes the number of cells and blocks of the structured grids for the simulations of the internal and external gas phase flows and the heat transfer in the solid strut material. The grids for the gas flow simulations are strongly refined near the walls $\left(y^{+} \approx 1\right)$ in order to meet the demands of the $q-\omega$ low-Reynolds number turbulence model on the one hand and to allow an accurate simulation of the heat transfer in the laminar sublayer on the other hand. Refinement is also applied to the mesh of the solid strut close to its inner and outer surfaces. The large number of 327 blocks required to mesh the solid strut is due to its complex internal and external shape. In consequence of this geometrical complexity of the injector and diverse additional requirements of the three individual grids (heat transfer in the solid, internal and external gas phase flow) the meshes do not coincide at the strut injector surfaces. Thus, the heat flux and wall temperature information is interpolated to the neighboring mesh for data exchange between the gas phase and the solid.

Table IV. Grid data for the solid strut and the internal and external gas phase flow simulations.

\begin{tabular}{lccc}
\hline Number of & strut & internal & external \\
\hline blocks & 327 & 54 & 82 \\
cells (million) & 0.417 & 1.568 & 4.878 \\
\hline
\end{tabular}




\subsection{Coupling of the different simulations}

Fig. 10 shows a typical plot of the maximum strut temperature over the number of simulation cycles. One simulation cycle consists of a predefined number of iterations with every solver (internal flow $\approx 10000$, external flow $\approx 30000$, and solid $\approx 90000$ iterations) after which heat flux data is exchanged. The convergence to a steady-state is accelerated by linear extrapolation of the temperature distributions and flow fields from consecutive simulation cycles. Although only two extrapolation steps are performed in this example (see Fig. 10), after ten cycles the maximum strut temperature changes by less than $1.0 \mathrm{~K}$ per cycle. On the contrary, for a simulation without extrapolation (and with uniform strut temperature initialization) more than 40 cycles are needed [46] to reach the same level of convergence $\left(\Delta T_{\max }<1.0 \mathrm{~K} /\right.$ cycle). The data extrapolation and an appropriate initialization significantly reduce the overall number of simulation cycles required. In the present case the simulation has been initialized with the converged solution from a similar injector configuration. Thus, only few simulation cycles are necessary to reach a steady-state solution.

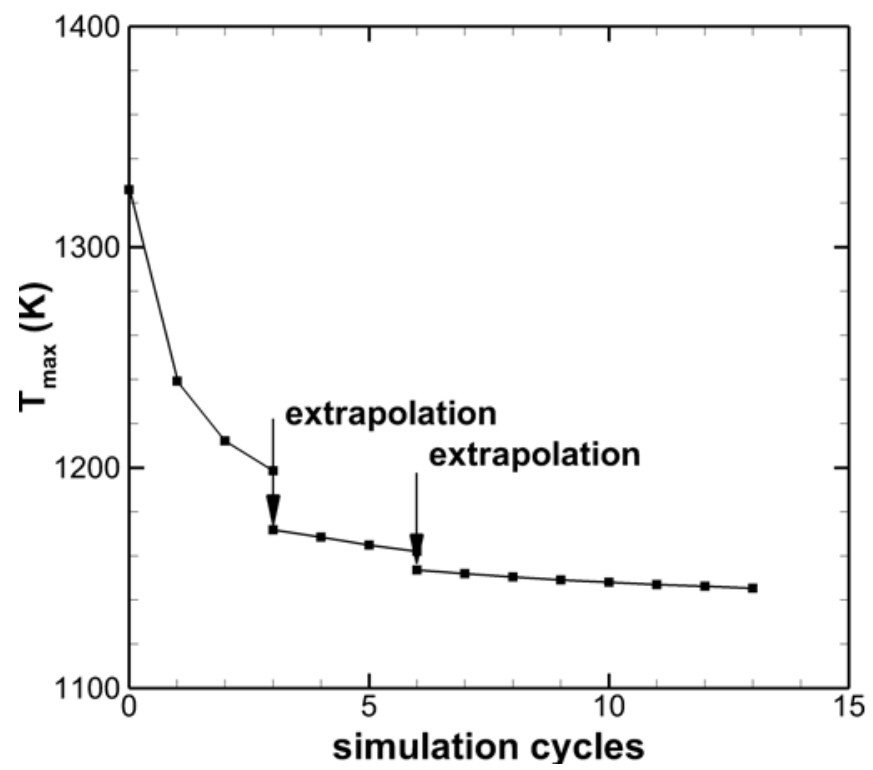

Figure 10. Maximum temperature of the strut injector versus the number of simulation cycles (data exchanges) with two steps of linear extrapolation for convergence acceleration. 


\subsection{External flow}

The Mach number, pressure and temperature distributions at the symmetry plane and at several cross sections of the combustor are shown in Fig. 11. The blunt tip of the injector causes a bow shock which is located approximately $1.75 \mathrm{~mm}$ upstream of the leading edge. Directly behind the shock a maximum pressure of about $p_{\max }=11 \mathrm{bar}$ and a maximum temperature of about $T_{\max }=2550 \mathrm{~K}$ are reached. Apart from the small region behind the bow shock and the boundary layers at the walls the flow remains supersonic in the whole combustor section. The normal shock wave at the tip quickly transitions to two oblique shock waves. Downstream, they are first reflected at the top and bottom walls of the combustor (at $x \approx 32.8 \mathrm{~mm}$ ). Then they cross the expansion fans, which are generated by the change in flow direction at the beginning of the injector ramps (see Mach number distribution in Fig. 11). Farther downstream the shock waves are reflected at the middle of the injector ramps $(x \approx 62-64 \mathrm{~mm})$, where a second hot region with temperatures above $1800 \mathrm{~K}$ is induced. The ramps generate pairs of counter-rotating streamwise vortices that cause a roll-up of the injected cold hydrogen layers and enhance the mixing with air [13]. This effect can be observed in the Mach number and temperature distributions of the cross sections downstream of the strut.

\subsection{Internal flow}

Figure 12 shows the internal Mach number (top) and temperature distributions (bottom) at the midplane for one half of the injector, respectively (please note that in the ramps this cut is through the midplane between the upper and lower walls). For such a complex flow field with many recirculation zones it is advantageous to use a high order discretization as demonstrated in the lid driven cavity test case in sect. 4.1.2. The fourth order MLP ${ }^{l d}$ discretization is a good compromise between accuracy and numerical stability which is a second important factor. The sixth order central discretization with a small amount of fifth order MLP discretization was not stable enough for this test case. The fifth order MLP scheme would have worked as well but for the external supersonic flow the stronger upwind character of the fourth order scheme is advantageous and the differences between fourth 

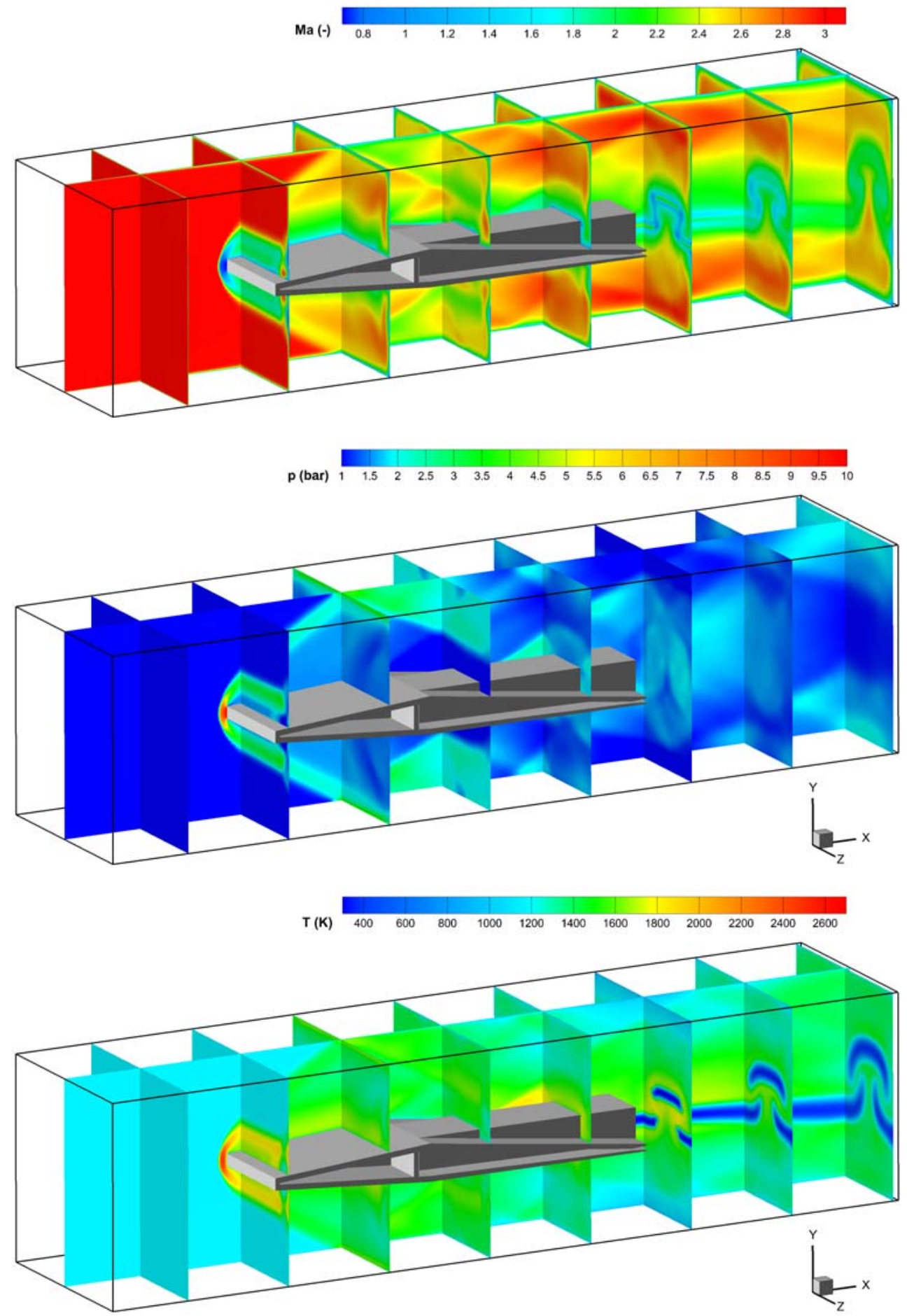

Figure 11. Mach number (top), pressure (center) and temperature distribution (bottom) at the symmetry plane and several equidistant cross sections $(\Delta x=0.02 \mathrm{~m})$. 
and fifth order MLP usually are very small. In this way both the internal and external flow fields could be simulated with the same type of discretization.

Although the flow inside the strut is accelerated in the nozzles at the end of the ramps to supersonic speed ( $M a \approx 2.25$ at the exit), a large portion of the internal flow is incompressible $(M a<0.3)$. Note that the shown distributions are located at the middle plane of the strut and that the flow velocities reduce approaching the upper or lower walls. Therefore an all-Mach number preconditioning (see subsection 2.1) is indispensable. The streamlines that are projected on the midplane in Fig. 12 visualize the complex flow patterns inside the strut injector. The hydrogen, which enters the strut through the side walls, is clearly directed towards the hot tip of the injector by the internal flow management. On its way to the ramps several small and large eddies cause a high level of turbulence increasing the heat transfer rate. Particularly the large zones of recirculation in the center of the strut extend the residence time of the hydrogen and cause an increased heat absorption. The average hydrogen temperature (mass flux averaged over all ramps) at the exit of the injector is 252.1 K. The average, the minimum and maximum hydrogen temperatures and the mass flux rates of each individual ramp are listed in Tab. V. Because of the proximity to the cool combustor side walls the lowest average hydrogen temperatures are found in ramps 1 and 5. Ramp 3 offers the most direct way for the hydrogen to flow through the strut. On its way to and through this ramp the flow is the least affected by eddies. Therefore the minimum and average temperatures at the exit of this ramp are lower than in ramp 2 and 4 . Significant temperature differences between the cold hydrogen core and the hot wall boundary layer cause a large spectrum of temperature levels at each ramp's exit. Despite the large eddies at the beginning of the ramps, the flow fields in the rear parts are very uniform and the mass flux distribution between the different ramps is relatively homogeneous. Ramp 3 shows the highest mass flux of all ramps: it differs by $+8.0 \%$ from a completely uniform mass flux distribution (which is $25 \%$ of the total mass flux for ramps $2-4$ and $12.5 \%$ for ramps 1 and 5, which are half as wide), respectively. 


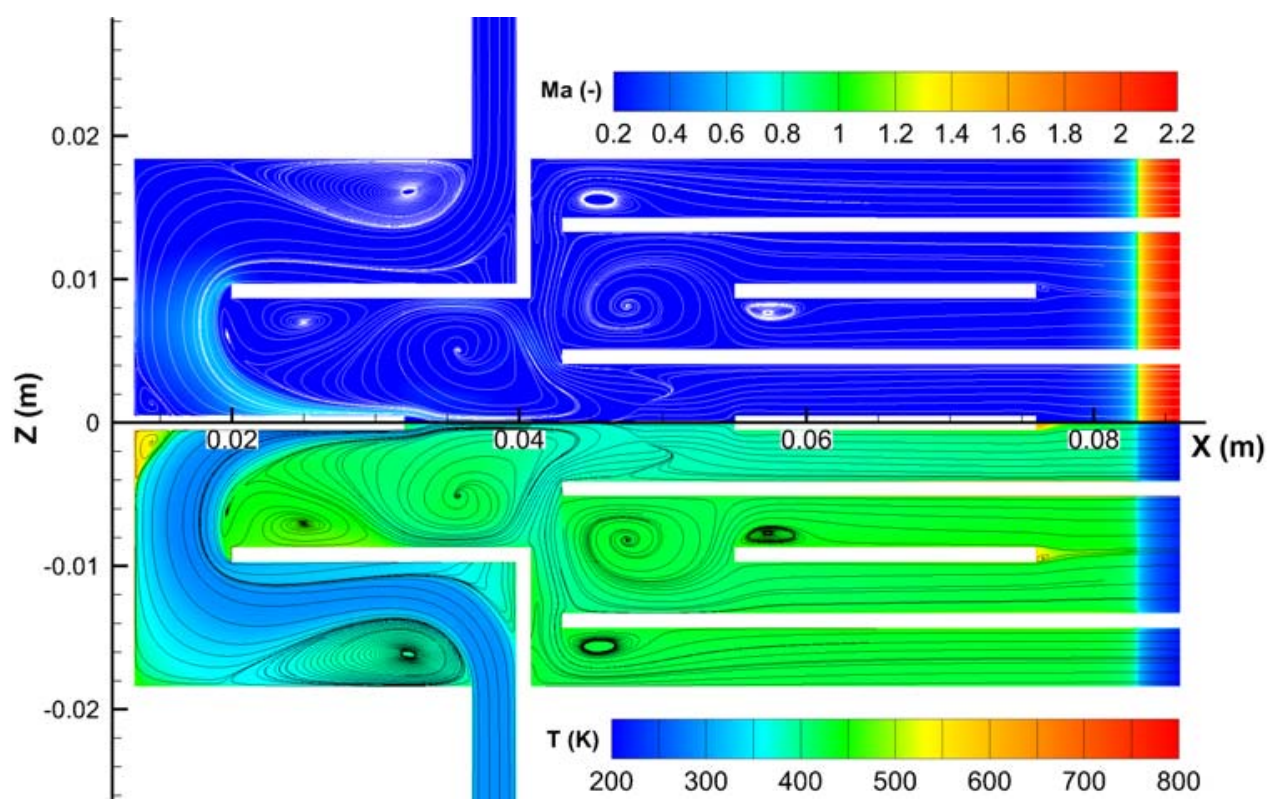

Figure 12. Distributions of Mach number (top) and temperature (bottom) at the midplane of the internal flow with streamlines.

Table V. Average, minimum and maximum hydrogen temperatures and relative mass fluxes at the exit of the different ramps.

\begin{tabular}{lccc}
\hline & ramps 1, 5 & ramps 2, 4 & ramp 3 \\
\hline $\bar{T}(\mathrm{~K})$ & 241.2 & 261.0 & 245.2 \\
$T_{\min }(\mathrm{K})$ & 207.9 & 213.4 & 201.5 \\
$T_{\max }(\mathrm{K})$ & 789.3 & 856.0 & 853.9 \\
$\dot{m}_{H_{2}}(\%)$ & 12.3 & 24.2 & 27.0 \\
\hline
\end{tabular}

\subsection{Solid strut}

The surface temperature of the solid strut is plotted in Fig. 13 with white isolines representing $T=$ $800 \mathrm{~K}$. The relatively cold combustor side walls significantly influence the temperature distribution in the injector material. The bow shock upstream of the strut charges the tip with high thermal loads. Nevertheless the hot temperature region at the tip with temperatures up to $818 \mathrm{~K}$ is remarkably small due to the efficient internal cooling of the strut. The maximum strut temperature of $880 \mathrm{~K}$ is located at the trailing edge of the strut where a second hot region results from the reflected oblique shock waves that hit the strut's ramps. In view of the melting temperature of copper of about $1350 \mathrm{~K}$ [25], the used hydrogen mass flux corresponding to an equivalence ratio of $\Phi=0.72$ satisfies the cooling requirements of the strut. The thermal interaction between the three domains may be seen in Fig. 14 


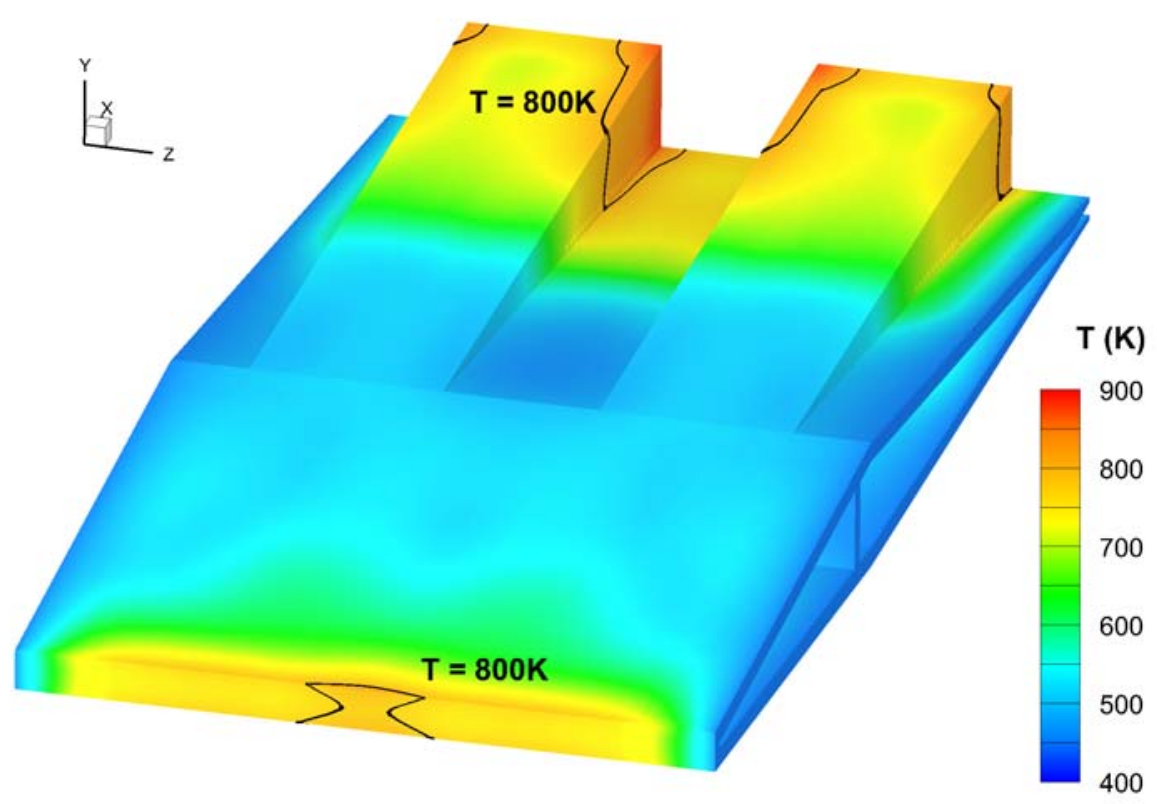

Figure 13. Temperature distribution at strut outer surface with isolines $T=800 \mathrm{~K}$ (black).

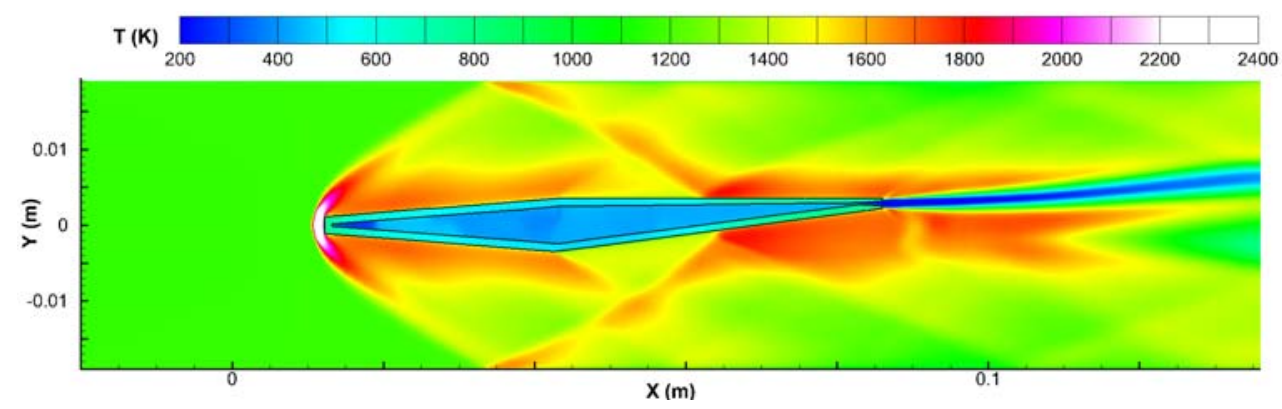

Figure 14. Temperature distribution at plane $Z=7 \mathrm{~mm}$ in the solid strut, the internal and external gas flows.

where the temperature distributions of the internal and external flows and the solid strut at the plane $Z=7 \mathrm{~mm}$ are combined in one plot. It shows the small region of particularly high gas temperatures of about $T_{\max }=2550 \mathrm{~K}$ in front of the tip of the strut, a layer of elevated gas temperatures around the front half of the strut that is diminished by the expansion fans at the beginning of the ramps, and finally a reincrease in temperature downstream of the reflected shock waves. Inside the strut particularly low hydrogen temperatures are situated in the region of the well cooled tip as well as at the end of the ramps where the temperature decreases due to the flow expansion in the nozzles. The temperature distribution at the trailing edge of the injector is plotted in Fig. 15 for the hydrogen flow 


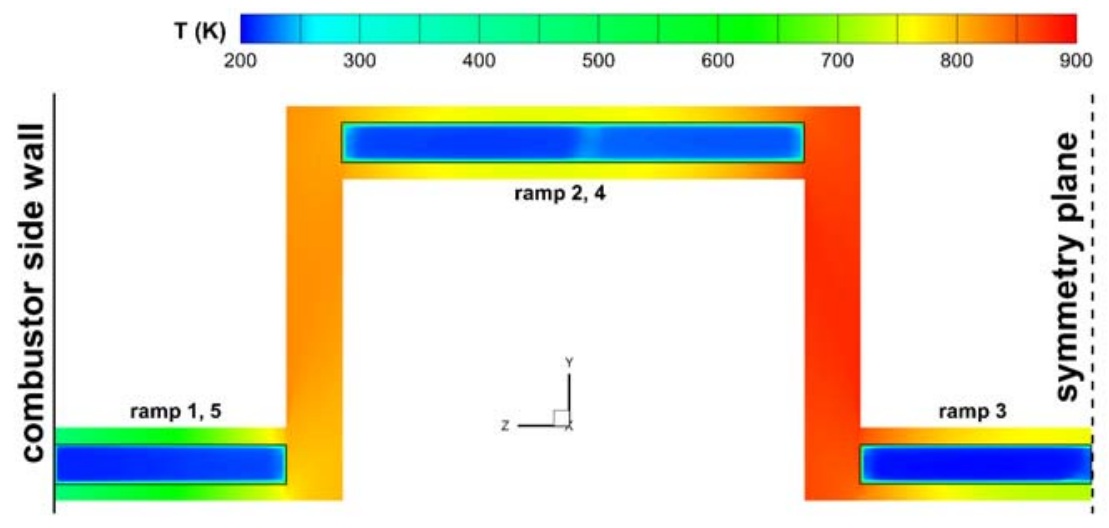

Figure 15. Temperature distribution in the solid strut and the hydrogen flow at the trailing edge (for one half of the injector).

and the solid material (please note that the left half of the strut up to the symmetry plane is shown only). Especially the vertical walls connecting ramp 3 with ramp 2 or 4, respectively, are heated by the reflected shock waves up to $880 \mathrm{~K}$. These vertical walls neither remarkably benefit from the cold combustor side walls (as they are too far away), nor from direct internal cooling (because only the horizontal parts of the ramps are internally cooled). Figure 15 also visualizes the hydrogen temperature differences within each ramp as well as inequalities between the different ramps, which have been discussed in the last subsection. An important information is the strong temperature difference in the injected hydrogen jet. Usually the strut internal flow is not simulated and the hydrogen is injected with a uniform temperature. As Fig. 15 shows, there are significant differences between the cold core (approximately $220 \mathrm{~K}$ ) and the hot boundary layer with temperatures higher than $700 \mathrm{~K}$. This may have an impact on mixing, ignition, and combustion. The heat flux from the external gas phase flow to the solid material of the strut injector is $\dot{Q}_{\mathrm{es}}=26.0 \mathrm{~kW}$. A heat flux of $\dot{Q}_{\mathrm{si}}=20.4 \mathrm{~kW}$ is absorbed by the internal hydrogen flow. Thus $78.5 \%$ of $\dot{Q}_{\mathrm{es}}$ are recovered by heating the later injected hydrogen. The remaining heat flux $(21.5 \%)$ is absorbed by the combustor side walls. Thus, the assumed constant wall temperature of $T_{\text {wall }}=450 \mathrm{~K}$ strongly contributes to the cooling of the strut injector at the investigated conditions. In general it may be stated, that the proposed internal flow management works successfully and that large parts of the heat losses from the main flow to the strut are recovered as the heated fuel is used for combustion. Moreover, the 
investigated equivalence ratio of $\Phi=0.72$ is suitable for the studied flight conditions at Mach 8 . The resulting maximum strut temperature undermatches the melting point of copper by about $470 \mathrm{~K}$.

\section{CONCLUSIONS}

The cooling of a lobed strut injector for scramjet applications is studied by a coupled simulation which combines the internal and external flow fields with the heat conduction in the solid material of the injector. For the internal mainly subsonic hydrogen flow a preconditioning technique is implemented and validated. The complex coupled simulation enables a complete and detailed analysis of the two gas phase flow fields, the temperature distribution in the solid injector body, interactions concerning heat transfer and the resulting heat fluxes. The major findings are:

1. The high order MLP $^{l d}$ discretization technique used here for the first time for a geometrical and numerical demanding simulation works reliably and stable and achieves excellent results. The additional computational cost is very low.

2. An extrapolation of data exchanged between the different solvers strongly reduces the number of couplings required to obtain a steady-state result.

3. The strategy to use the fuel for active cooling is a promising concept, capable to sufficiently cool strut injectors in scramjet combustors for flight conditions.

4. The coupled simulations give insight into realistic hydrogen outflow conditions at the exit of the strut injector. They can be used as boundary conditions for future scramjet combustor studies without internal hydrogen flow simulation.

In summary the proposed numerical technique for strut injector simulation is a suited tool for scramjet design.

\section{ACKNOWLEDGEMENTS}

This work has been performed within the GRK 1095/2 project investigating the "Aero-Thermodynamic Design of a Scramjet Engine for Future Space Transportation Systems". It has been supported by the 
Deutsche Forschungsgemeinschaft (DFG). We also wish to thank M. Lourier for providing the heat flux code and the High Performance Computing Center Stuttgart (HLRS) for providing computational resources.

\section{REFERENCES}

1. Yoon SH, Kim C, Kim KH. Multi-Dimensional Limiting Process for Three-Dimensional Flow Physics Analyses. Journal of Computational Physics 2008; 227:pp. 6001-6043.

2. Gerlinger P. Multi-Dimensional Limiting for High-Order Schemes Including Turbulence and Combustion. Journal of Computational Physics 2012; 231(5):pp. 2199-2228.

3. Kodera M, Sunami T, Itoh K. Numerical Simulation of a Scramjet Engine for JAXAs Flight Experiment Using HyShot. AIAA 2005-3335, 2005.

4. Schramm JM, Karl S, Hannemann K, Steelant J. Ground Testing of the HyShot II Scramjet Configuration in HEG. AIAA 2008-2547, 2008.

5. Smart MK, Suraweera MV. HIFiRE 7 - Development of a 3D Scramjet for Flight Testing. AIAA 2009-7259, 2009.

6. Waitz I, Marble F, Zukoski E. Investigation of a Contoured Wall Injector for Hypervelocity Mixing Augmentation. AIAA Journal 1993; 31:1014-1021.

7. Baurle R, Fuller R, White J, Chen T, Gruber M, Nejad A. An Investigation of Advanced Fuel Injection Schemes for Scramjet Combustion. AIAA 98-0937, 1998.

8. Glamwe D, Samimiy M, Nejad A, Cheng T. Effects of Nozzle Geometry on Parallel Injection from Base of an Extended Strut into Supersonic Flow. AIAA 95-0522, 1995.

9. Strickland J, Selerland T, Karagozian AR. Numerical Simulation of a Lobed Fuel Injector. Journal of Propulsion and Power 1998; 10:pp. 2950-2964.

10. Sunami T, Wendt M, Nishioka M. Supersonic Mixing and Combustion Control using Streamwise Vorticity. AIAA 98-3271, 1998.

11. Simsont YH, Gerlinger P. Numerical Investigation of a Complete Scramjet Model with Central Strut Injection. ISABE Paper 2013-1619, 2013.

12. Gerlinger P, Kasal P, Stoll P, Brüggemann D. Experimental and Numerical Investigation of 2D and 3D Parallel Hydrogen/Air Mixing in Supersonic Flows. ISABE Paper 2001-1019, 2001.

13. Gerlinger P, Stoll P, Kindler M, Schneider F, Aigner M. Numerical Investigation of Mixing and Combustion Enhancement in Supersonic Combustors by Strut Induced Streamwise Vorticity. Aerospace Science and Technology 2008; 12:pp. 159-168.

14. Kindler M, Gerlinger P, Aigner M. Hybrid RANS/LES of Lobed Strut Injectors in Supersonic Flow. ISABE Paper 2013-1625, 2013.

15. Kuaran K, Behera PR, Babu V. Numerical Investigation of Supersonic Combustion of Kerosene in a Strut-Based Combustor. Journal of Propulsion and Power 2010; 26:pp. 1084-1091. 
16. Sujith S, Muruganandam T, Kurian J. Effect of Trailing Ramp Angles in Strut-Based Injection in Supersonic Flow. Journal of Propulsion and Power 2013; 29:pp. 66-78.

17. Lee SH. Mixing Augmentation with Cooled Pylon Injection in a Scramjet Combustor. Journal of Propulsion and Power 2012; 28:pp. 477-485.

18. Gerlinger P, Kasal P, Schneider F, von Wolfersdorf J, Aigner M. Experimental and Numerical Investigation of Lobed Strut Injectors for Supersonic Combustion. D. Jacob, G. Sachs, S. Wagner (Edts.), Basic Research and Technologies for Two-Stage-to-Orbit Vehicles, Wiley-VCH Verlag GmbH \& Co. HGaG, 2005.

19. Banica MC, Scheuermann T, Chun J, Weigand B, von Wolfersdorf J. Numerical Study of Supersonic Combustion Process with Central Strut Injection. Journal of Propulsion and Power 2010; 26:pp. 869-874.

20. Kim KH, Kim C. Accurate, Efficient and Monotonic Numerical Methods for Multi-Dimenional Compressible Flows - Part II: Multi-Dimensional Limiting Process. Journal of Computational Physics 2005; 208:pp. 570-615.

21. Gerlinger P, Brüggemann D. Multigrid Convergence Acceleration for Turbulent Supersonic Flows. International Journal for Numerical Methods in Fluids 1997; 24:pp. 1019-1035.

22. Gerlinger P, Möbus H, Brüggemann D. An Implicit Multigrid Method for Turbulent Combustion. Journal of Computational Physics 2001; 167:pp. 247-276.

23. Lourier JM. Numerische Untersuchung zum konjugierten Wärmeübergang in Scramjet-Brennkammern. Diploma Thesis, RWTH Aachen University, 2009.

24. Coakley TJ, Huang PG. Turbulence Modeling for High Speed Flows. AIAA 92-0436, 1992.

25. Çengel YA. Heat and Mass Transfer: A Practical Approach. 3rd Edition. McGraw-Hill Science Engineering/Math, 2006.

26. Jameson A, Yoon S. Lower-Upper Implicit Scheme with Multiple Grids for the Euler Equations. AIAA Journal 1987; 25:pp. 929-937.

27. Shuen JS. Upwind Differencing and LU Factorization for Chemical Non-Equilibrium Navier-Stokes Equations. Journal of Computational Physics 1992; 99:pp. 233-250.

28. Gerlinger P, Brüggemann D. An Implicit Multigrid Scheme for the Compressible Navier-Stokes Equations with Low-Reynolds-Number Turbulence Closure. Journal of Fluids Engineering 1998; 120:pp. 257-262.

29. Liou MS. A Sequel to AUSM - Part II: $\mathrm{AUSM}^{+}$-up for all Speeds. Journal of Computational Physics 2006; 214:pp. $137-170$.

30. Stoll P, Gerlinger P, Brüggemann D. Domain Decomposition for an Implicit LU-SGS Scheme using Overlapping Grids. AIAA 97-1869, 1997

31. Jameson A. Time Dependent Calculations Using Multigrid with Applications to Unsteady Flows Past Airfoils and Wings. AIAA 91-1596, 1991.

32. Arnone A, Liou MS, Povinelli LA. Multigrid Time-Accurate Integration of Navier-Stokes Equations. AIAA 933361, 1993.

33. Choi YH, Merkle CL. The Application of Preconditioning in Viscous Flows. AIAA Journal 1993; 105:pp. $207-223$. 
34. Sweby PK. High Resolution Schemes using Flux Limiters for Hyperbolic ConservationLlaws. SIAM Journal on Numerical Analysis 1984; 21:pp. 995-1011.

35. Kindler M, Gerlinger P, Aigner M. Investigation of Hybrid rans/les Approaches for Compressible High Speed Flows. AIAA 2011-2218, 2011.

36. Douglas J, Gunn JE. A General Formulation of Alternating Direction Methods - Part I: Parabolic and Hyperbolic Problems. Journal of Numerical Mathematics 1964; 6:pp. 428-453.

37. Thomas LH. Elliptic Problems in Linear Differential Equations over a Network. Watson Scientific Computing Laboratory Report, Columbia University, New York, 1949.

38. Tannehill JC, Anderson DA, Pletcher RH. Computational Fluid Mechanics and Heat Transfer, Second Edition. McGraw Hill Higher Education, 1984.

39. Gerlinger P, Stoll P, Brüggemann D. An Implicit Multigrid Method for the Simulation of Chemically Reacting Flows. Journal of Computational Physics 1998; 146:pp. 322-345.

40. Gerlinger P, Aigner M. Multigrid Simulations of Detached Shock Waves. International Journal for Numerical Methods in Fluids 2004; 44:pp. 1045-1061.

41. Ghia U, Ghia KN, Shin CT. High-Re Solutions for Incompressible Flow Using the Navier-Stokes Equations and a Multigrid Method. Journal of Computational Physics 1982; 48:pp. 387-411.

42. Kasal P, Gerlinger P, Walther R, von Wolfersdorf J, Weigand B. Supersonic Combustion: Fundamental Investigation of Aerothermodynamic Key Problems. AIAA 2002-5119, 2002.

43. Kindler M, Gerlinger P, Aigner M. Numerical Investigation of Mixing Enhancement by Lobed Strut Injectors in Turbulent Reactive Supersonic Flow. ISABE Paper 2007-1314, 2007.

44. Rust B, Gerlinger P, Aigner M. An Improved Lobed Strut Injector Concept for Supersonic Combustion. AIAA 2010-6062, 2010.

45. Gerlinger P, Schneider F, Aigner M. Numerical Investigation of Mixing Enhancement by Streamwise Vorticity in Supersonic Combustors. ISABE Paper 2005-1019, 2005.

46. Gerlinger P, Simsont YH. Numerical Simulation of the Internal and External Flow Fields and Heat Fluxes of a Lobed Strut Injector. ISABE Paper 2013-1627, 2013. 